\title{
Nonlinear dynamic response and vibration of imperfect shear deformable functionally graded plates subjected to blast and thermal loads
}

\author{
Nguyen Dinh Duc, Ngo Duc Tuan, Phuong Tran \& Tran Quoc Quan
}

To cite this article: Nguyen Dinh Duc, Ngo Duc Tuan, Phuong Tran \& Tran Quoc Quan (2016): Nonlinear dynamic response and vibration of imperfect shear deformable functionally graded plates subjected to blast and thermal loads, Mechanics of Advanced Materials and Structures, DOI: $10.1080 / 15376494.2016 .1142024$

To link to this article: http://dx.doi.org/10.1080/15376494.2016.1142024

Accepted author version posted online: 29

Jan 2016.

Submit your article to this journal $\llbracket$

Џll Article views: 17

View related articles $₫$

View Crossmark data $\nearrow$ 


\title{
ACCEPTED MANUSCRIPT
}

\section{Nonlinear dynamic response and vibration of imperfect shear deformable functionally}

\author{
graded plates subjected to blast and thermal loads
}

\author{
Nguyen Dinh Duc ${ }^{1 *}$, Ngo Duc Tuan ${ }^{2}$, Phuong Tran ${ }^{2}$, Tran Quoc Quan ${ }^{1}$ \\ ${ }^{1}$ Vietnam National University, Hanoi -144 Xuan Thuy - Cau Giay - Hanoi - Vietnam \\ ${ }^{2}$ The University of Melbourne, Parkville 3010, VIC, Australia
}

Received: 2015-06-09

Accepted: 2015-12-23

* Corresponding author: Email: ducnd@ vnu.edu.vn (Duc ND)

\begin{abstract}
Based on Reddy's higher-order shear deformation plate theory, this paper presents an analysis of the nonlinear dynamic response and vibration of imperfect functionally graded material (FGM) thick plates subjected to blast and thermal loads resting on elastic foundations. The material properties are assumed to be temperature-dependent and graded in the thickness direction according to a simple power-law distribution in terms of the volume fractions of the constituents. Numerical results for the dynamic response and vibration of the FGM plates with two cases of boundary conditions are obtained by the Galerkin method and fourth-order Runge-Kutta method. The results show the effects of geometrical parameters, material properties, imperfections, temperature increment, elastic foundations and boundary conditions on the nonlinear dynamic response and vibration of FGM plates.
\end{abstract}

Keywords: Nonlinear dynamic response, vibration, FGM plates, higher-order shear deformation plate theory, blast and thermal loads, elastic foundations. 


\section{ACCEPTED MANUSCRIPT}

\section{Introduction}

The concept of functionally graded materials (FGMs) was first introduced in Japan in 1984 during a space plane project. FGMs are microscopically inhomogeneous materials that exhibit continuous variation of material properties from one surface to another and thus eliminate the stress concentration generally found in conventional laminated composites. FGM structures are widely used in various engineering applications such as hoppers, marine and ocean engineering structures, components of missiles and spacecraft, and other civil applications.

Therefore, the problems of static and dynamic stability of structures such as FGM plates and shells have attracted increasing research effort. Asemi et al. [1] investigated the shear buckling analysis of FGM annular sector plates based on a three-dimensional elasticity approach. Nejad et al. [2] introduced a semi-analytical solution for the purpose of elastic analysis of rotating thick FGM truncated conical shells under non-uniform pressure; whilst Dozio [3] derived the firstknown exact solutions for free vibration of thick and moderately-thick FGM rectangular plates on the basis of a family of two-dimensional shear and normal deformation theories with variable order. Duc [4] investigated the nonlinear dynamic response of imperfect eccentrically stiffened FGM double curved shallow shells on elastic foundations. Alipour and Shariyat [5] studied the stress and deformation analysis of FGM annular sandwich plates subjected to non-uniform normal and/or shear tractions. Duc et al. [6] considered the nonlinear dynamic analysis and vibration of imperfect FGM thick doubly curved shallow shells with piezoelectric actuators on 


\section{ACCEPTED MANUSCRIPT}

elastic foundations subjected to the combination of electrical, thermal, mechanical and damping loading; and Shen [7] analysed thermal post-buckling analysis for a simply supported, shear deformable functionally graded plate under thermal loading. Dai et al. [8] presented an analysis of vibrations and transient responses of FGM hollow cylinder under a radially symmetric dynamic load. Loc et al. [9] proposed a novel and effective formulation that combines the extended isogeometric approach and higher-order shear deformation theory to study the free vibration of cracked FGM plates. Huang and Han [10] investigated the nonlinear dynamic buckling problems of unstiffened FGM cylindrical shells subjected to time-dependent axial load by using a one-term solution form. In 2014, Duc [11] published a valuable book "Nonlinear static and dynamic stability of functionally graded plates and shells", in which the results of nonlinear dynamic analysis of shear deformable FGM structures were presented.

In recent years, buildings and critical infrastructures across the globe have become more vulnerable to extreme dynamic explosion and impact loads due to increased terrorist activities, accidental explosions, proliferation of weapons, and so forth. As a result, blast loads and their impact on the safety and performance of structures have received considerable interest. Shi et al. [12] conducted a spatial reliability analysis to predict the damage on reinforced concrete columns subjected to explosive blast loading. Gauch et al. [13] presented the effect of preloading a thin composite plate subjected to underwater explosive loading through computational simulations; and Ignatieva et al. [14] investigated the peculiarities of the structure of copper- and nickel-fluoropolymer composites fabricated by explosive pressing. Jones [15] carried out tests on the dynamic 


\section{ACCEPTED MANUSCRIPT}

inelastic response of strain rate sensitive ductile plates due to large impact, dynamic pressure and explosive loadings. Mohotti et al. [16] conducted an experimental and numerical study to determine the deformation time history behaviour of square aluminium alloy AA5083-H116 plates supported by a bolted frame subjected to low velocity impact. Yehia et al. [17] investigated wave propagation and dispersion in sandwich plates subjected to blast loads. Further, Ackland et al. [18] performed experimental and numerical studies to investigate the effect of polyurea coatings on the blast resistance of mild steel plates. Gonçalves et al. [19] researched the impact of projectiles on ceramic/metal armour using a simple one-dimensional mode. Luong et al. [20] presented an analysis of the underground structure in a coral foundation under dynamic loading; and Geretto et al. [21] dealt with the experimental analysis of square mild steel plates subjected to blast loads in three different degrees of confinement. The energy absorption of circular and square aluminium alloy tubes subjected to an axial explosive load, which is transmitted to a tube by a small attached mass, is discussed in the investigation of Karagiozova et al. [22].

To the authors' best knowledge, there are very little researches on the problems of FGM structures subjected to blast loads. Aksoylar et al. [23] studied the nonlinear transient analysis of FGM and fibre-metal laminated plates under blast loads by experimental and mixed finite element methods. Hause [24] developed the foundation of the theory of FGM plates with simply supported edges, under a Friedlander explosive air-blast within the classical plate theory. Further, Übeyli et al. [25] investigated the potential of using silicon carbide reinforced FGM as 


\section{ACCEPTED MANUSCRIPT}

armour material when impacted by an armour-piercing projectile. Lam et al. [26] developed the modelling of blast pressure for engineering applications. Bodaghi et al. [27] presented nonlinear active control of the dynamic response of FGM beams with rectangular cross-sections in thermal environments exposed to blast loadings. To date, no studies on the analytical approach to investigate the dynamic response of FGM plates subjected to blast load has been published.

New contribution of the paper is that this is the first investigation successfully establish modeling and analytical formulations for the nonlinear dynamic response and vibration of FGM thick plates subjected to blast and thermal loads using the higher-order shear deformation plate theory. Material properties are assumed to be temperature-dependent. Two cases of boundary conditions are considered. The Galerkin method and fourth-order Runge-Kutta method are used to solve basic equations.

\section{Problem statement}

Consider an FGM plate on elastic foundations as shown in Figure 1. A coordinate system $(x, y, z)$ is established, in which the $(x, y)$ plane is on the middle surface of the plate and $z$ is the thickness direction $(-h / 2 \leq z \leq h / 2)$. The length, width, and total thickness of the plate are $a, b$ and $h$, respectively.

By applying the power-law distribution, the volume fractions of ceramic and metal are assumed as:

$$
V_{c}(z)=\left(\frac{2 z+h}{2 h}\right)^{N}, V_{m}(z)=1-V_{c}(z),
$$




\section{ACCEPTED MANUSCRIPT}

where $N$ is the volume fraction index $(0 \leq N<\infty)$; and subscripts $m$ and $c$ stand for the metal and ceramic constituents, respectively. The effective properties, $P r_{\text {eff }}$, of the FGM plate, such as the elastic modulus $E$, the mass density $\rho$, and the thermal expansion coefficient $\alpha$ are determined by a linear rule of mixture as:

$$
\operatorname{Pr}_{\mathrm{eff}}(z)=\operatorname{Pr}_{c} V_{c}(z)+\operatorname{Pr}_{m} V_{m}(z)
$$

in which $\operatorname{Pr}$ denotes a temperature-dependent material property. The effective properties of the FGM plate are obtained by substituting Eq. (1) into Eq. (2) as:

$$
[E(z), \rho(z), \alpha(z)]=\left[E_{m}, \rho_{m}, \alpha_{m}\right]+\left[E_{c m}, \rho_{c m}, \alpha_{c m}\right]\left(\frac{2 z+h}{2 h}\right)^{N},
$$

where,

$$
E_{c m}=E_{c}-E_{m}, \rho_{c m}=\rho_{c}-\rho_{m}, \alpha_{c m}=\alpha_{c}-\alpha_{m},
$$

and Poisson's ratio is assumed to be constant, $v(z)=v=$ const .

A material property, $P r$, can be expressed as a nonlinear function of temperature $[6,7$, 11]:

$$
\operatorname{Pr}=P_{0}\left(P_{-1} T^{-1}+1+P_{1} T+P_{2} T^{2}+P_{3} T^{3}\right),
$$

in which $T=T_{0}+\Delta T, \Delta T$ is the temperature increment in the environment containing the panel and $T_{0}=300 \mathrm{~K}$ (room temperature), and $P_{0}, P_{-1}, P_{1}, P_{2}$ and $P_{3}$ are coefficients characterizing the constituent materials.

The plate-foundation interaction of the Pasternak model is given by: 


\section{ACCEPTED MANUSCRIPT}

$$
q_{e}=k_{1} w-k_{2} \nabla^{2} w
$$

in which $\nabla^{2}=\partial^{2} / \partial x^{2}+\partial^{2} / \partial y^{2}, w$ is the deflection of the plate, $k_{1}$ is the Winkler foundation modulus, and $k_{2}$ is the shear layer foundation stiffness of the Pasternak model.

\section{Governing equations}

In the present study, higher-order shear deformation plate theory is used to derive the governing equations and determine the nonlinear response of FGM thick plates.

The strain components across the plate thickness at a distance $z$ from the mid-plane are represented by:

$$
\begin{gathered}
\left(\begin{array}{c}
\varepsilon_{x} \\
\varepsilon_{y} \\
\gamma_{x y}
\end{array}\right)=\left(\begin{array}{c}
\varepsilon_{x}^{0} \\
\varepsilon_{y}^{0} \\
\gamma_{x y}^{0}
\end{array}\right)+z\left(\begin{array}{l}
k_{x}^{1} \\
k_{y}^{1} \\
k_{x y}^{1}
\end{array}\right)+z^{3}\left(\begin{array}{l}
k_{x}^{3} \\
k_{y}^{3} \\
k_{x y}^{3}
\end{array}\right), \\
\left(\begin{array}{l}
\gamma_{x z} \\
\gamma_{y z}
\end{array}\right)=\left(\begin{array}{l}
\gamma_{x z}^{0} \\
\gamma_{y z}^{0}
\end{array}\right)+z^{2}\left(\begin{array}{l}
k_{x z}^{2} \\
k_{y z}^{2}
\end{array}\right),
\end{gathered}
$$

where,

$$
\begin{gathered}
\left(\begin{array}{c}
\varepsilon_{x}^{0} \\
\varepsilon_{y}^{0} \\
\gamma_{x y}^{0}
\end{array}\right)=\left(\begin{array}{c}
u_{, x}+w_{, x}^{2} / 2 \\
v_{, y}+w_{, y}^{2} / 2 \\
u_{, y}+v_{, x}+w_{, x} w_{, y}
\end{array}\right),\left(\begin{array}{c}
k_{x}^{1} \\
k_{y}^{1} \\
k_{x y}^{1}
\end{array}\right)=\left(\begin{array}{c}
\phi_{x, x} \\
\phi_{y, y} \\
\phi_{x, y}+\phi_{y, x}
\end{array}\right), \\
\left(\begin{array}{c}
k_{x}^{3} \\
k_{y}^{3} \\
k_{x y}^{3}
\end{array}\right)=-c_{1}\left(\begin{array}{c}
\phi_{x, x}+w_{, x x} \\
\phi_{y, y}+w_{, y y} \\
\phi_{x, y}+\phi_{y, x}+2 w_{, x y}
\end{array}\right),\left(\begin{array}{l}
\gamma_{x z}^{0} \\
\gamma_{y z}^{0}
\end{array}\right)=\left(\begin{array}{l}
\phi_{x}+w_{, x} \\
\phi_{y}+w_{, y}
\end{array}\right),\left(\begin{array}{l}
k_{x z}^{2} \\
k_{y z}^{2}
\end{array}\right)=-3 c_{1}\left(\begin{array}{l}
\phi_{x}+w_{, x} \\
\phi_{y}+w_{, y}
\end{array}\right),
\end{gathered}
$$




\section{ACCEPTED MANUSCRIPT}

in which $c_{1}=4 / 3 h^{2} ; u, v$ are the displacement components along the $x, y$ directions, respectively; and $\phi_{x}, \phi_{y}$ are the rotations of normals to the mid-surface with respect to the $x$ and $y$ axes, respectively.

Hooke's stress-strain relation is applied for the FGM plate as:

$$
\begin{aligned}
& \left(\sigma_{x}, \sigma_{y}\right)=\frac{E}{1-v^{2}}\left[\left(\varepsilon_{x}, \varepsilon_{y}\right)+v\left(\varepsilon_{y}, \varepsilon_{x}\right)-(1+v) \alpha \Delta T(1,1)\right], \\
& \left(\sigma_{x y}, \sigma_{x z}, \sigma_{y z}\right)=\frac{E}{2(1+v)}\left(\gamma_{x y}, \gamma_{x z}, \gamma_{y z}\right) .
\end{aligned}
$$

The force and moment resultants are expressed by:

$$
\begin{aligned}
& \left(N_{i}, M_{i}, P_{i}\right)=\int_{-h / 2}^{h / 2} \sigma_{i}\left(1, z, z^{3}\right) d z, i=x, y, x y, \\
& \left(Q_{i}, K_{i}\right)=\int_{-h / 2}^{h / 2} \sigma_{i z}\left(1, z^{2}\right) d z, i=x, y .
\end{aligned}
$$

Substituting Eqs. (7) into Eqs. (9), and the result into Eqs. (10) gives: 


\section{ACCEPTED MANUSCRIPT}

$$
\begin{aligned}
& \left(N_{x}, M_{x}, P_{x}\right)=\frac{1}{1-v^{2}}\left[\left(E_{1}, E_{2}, E_{4}\right)\left(\varepsilon_{x}^{0}+v \varepsilon_{y}^{0}\right)+\left(E_{2}, E_{3}, E_{5}\right)\left(k_{x}^{1}+v k_{y}^{1}\right)\right. \\
& \left.+\left(E_{4}, E_{5}, E_{7}\right)\left(k_{x}^{3}+v k_{y}^{3}\right)-(1+v)\left(\Phi_{1}, \Phi_{2}, \Phi_{4}\right)\right], \\
& \left(N_{y}, M_{y}, P_{y}\right)=\frac{1}{1-v^{2}}\left[\left(E_{1}, E_{2}, E_{4}\right)\left(\varepsilon_{y}^{0}+v \varepsilon_{x}^{0}\right)+\left(E_{2}, E_{3}, E_{5}\right)\left(k_{y}^{1}+v k_{x}^{1}\right)\right. \\
& \left.+\left(E_{4}, E_{5}, E_{7}\right)\left(k_{y}^{3}+v k_{x}^{3}\right)-(1+v)\left(\Phi_{1}, \Phi_{2}, \Phi_{4}\right)\right], \\
& \left(N_{x y}, M_{x y}, P_{x y}\right)=\frac{1}{2(1+v)}\left[\left(E_{1}, E_{2}, E_{4}\right) \gamma_{x y}^{0}+\left(E_{2}, E_{3}, E_{5}\right) k_{x y}^{1}+\left(E_{4}, E_{5}, E_{7}\right) k_{x y}^{3}\right], \\
& \left(Q_{x}, K_{x}\right)=\frac{1}{2(1+v)}\left[\left(E_{1}, E_{3}\right) \gamma_{x z}^{0}+\left(E_{3}, E_{5}\right) k_{x z}^{2}\right], \\
& \left(Q_{y}, K_{y}\right)=\frac{1}{2(1+v)}\left[\left(E_{1}, E_{3}\right) \gamma_{y z}^{0}+\left(E_{3}, E_{5}\right) k_{y z}^{2}\right],
\end{aligned}
$$

where,

$$
\begin{aligned}
& \left(E_{1}, E_{2}, E_{3}, E_{4}, E_{5}, E_{7}\right)=\int_{-h / 2}^{h / 2} E(z)\left(1, z, z^{2}, z^{3}, z^{4}, z^{6}\right) d z, \\
& \left(\Phi_{1}, \Phi_{2}, \Phi_{4}\right)=\int_{-h / 2}^{h / 2} E(z) \alpha(z) \Delta T\left(1, z, z^{3}\right) d z,
\end{aligned}
$$

The nonlinear equilibrium equations of a perfect FGM plate based on the higher-order shear deformation plate theory are [11]:

$$
\begin{aligned}
& N_{x, x}+N_{x y, y}=I_{1} \frac{\partial^{2} u}{\partial t^{2}}+\bar{I}_{2} \frac{\partial^{2} \phi_{x}}{\partial t^{2}}-\bar{I}_{3} \frac{\partial^{3} \mathrm{w}}{\partial t^{2} \partial x} \\
& N_{x y, x}+N_{y, y}=I_{1} \frac{\partial^{2} v}{\partial t^{2}}+\bar{I}_{2} \frac{\partial^{2} \phi_{y}}{\partial t^{2}}-\bar{I}_{3} \frac{\partial^{3} \mathrm{w}}{\partial t^{2} \partial y},
\end{aligned}
$$




\section{ACCEPTED MANUSCRIPT}

$$
\begin{gathered}
Q_{x, x}+Q_{y, y}-3 c_{1}\left(K_{x, x}+K_{y, y}\right)+c_{1}\left(P_{x, x x}+2 P_{x y, x y}+P_{y, y y}\right) \\
+N_{x} w_{, x x}+2 N_{x y} w_{, x y}+N_{y} w_{, y y}+p-k_{1} w+k_{2} \nabla^{2} w= \\
=I_{1} \frac{\partial^{2} w}{\partial t^{2}}+2 \varepsilon I_{1} \frac{\partial \mathrm{w}}{\partial t}+\bar{I}_{3}\left(\frac{\partial^{3} u}{\partial t^{2} \partial x}+\frac{\partial^{3} v}{\partial t^{2} \partial y}\right)+\bar{I}_{5}\left(\frac{\partial^{3} \phi_{x}}{\partial t^{2} \partial x}+\frac{\partial^{3} \phi_{y}}{\partial t^{2} \partial y}\right) \\
-c_{1}^{2} I_{7}\left(\frac{\partial^{4} \mathrm{w}}{\partial t^{2} \partial x^{2}}+\frac{\partial^{4} \mathrm{w}}{\partial t^{2} \partial y^{2}}\right), \\
M_{x, x}+M_{x y, y}-Q_{x}+3 c_{1} K_{x}-c_{1}\left(P_{x, x}+P_{x y, y}\right)=\overline{I_{2}} \frac{\partial^{2} u}{\partial t^{2}}+\overline{I_{4}} \frac{\partial^{2} \phi_{x}}{\partial t^{2}}-\overline{I_{5}} \frac{\partial^{3} \mathrm{w}}{\partial t^{2} \partial x}, \\
M_{x y, x}+M_{y, y}-Q_{y}+3 c_{1} K_{y}-c_{1}\left(P_{x y, x}+P_{y, y}\right)=\overline{I_{2}} \frac{\partial^{2} v}{\partial t^{2}}+\overline{I_{4}} \frac{\partial^{2} \phi_{y}}{\partial t^{2}}-\overline{I_{5}} \frac{\partial^{3} \mathrm{w}}{\partial t^{2} \partial y},
\end{gathered}
$$

where $\varepsilon$ is the damping coefficient, and

$$
\begin{aligned}
& \overline{I_{2}}=I_{2}-c_{1} I_{4}, \overline{I_{3}}=c_{1} I_{4}, \overline{I_{4}}=I_{3}-2 c_{1} I_{5}+c_{1}^{2} I_{7}, \overline{I_{5}}=c_{1} I_{5}-c_{1}^{2} I_{7}, \\
& \left(I_{1}, I_{2}, I_{3}, I_{4}, I_{5}, I_{7}\right)=\int_{-h / 2}^{h / 2} \rho(z)\left(1, z, z^{2}, z^{3}, z^{4}, z^{6}\right) d z .
\end{aligned}
$$

The blast load $p(t)$ is a short-term load and is generated by an explosion or by a shockwave disturbance produced by an aircraft flying at supersonic speed, or by a supersonic projectile, rocket or missile operating in its vicinity. It can be expressed as [26]:

$$
p(t)=1.8 P s_{\max }\left(1-\frac{t}{T_{s}}\right) \exp \left(\frac{-b t}{T_{s}}\right),
$$

where the "1.8" factor accounts for the effects of a hemispherical blast, $P s_{\max }$ is the maximum (or peak) static over-pressure, $b$ is the parameter controlling the rate of wave amplitude decay and $T_{s}$ is the parameter characterizing the duration of the blast pulse. 


\section{ACCEPTED MANUSCRIPT}

From the constitutive relations (11), one can inversely obtain:

$$
\begin{aligned}
& \left(\varepsilon_{x}^{0}, \varepsilon_{y}^{0}\right)=\frac{1}{E_{1}}\left[\left(f_{, y y}, f_{, x x}\right)-v\left(f_{, x x}, f_{, y y}\right)-E_{2}\left(k_{x}^{1}, k_{y}^{1}\right)-E_{4}\left(k_{x}^{3}, k_{y}^{3}\right)+\Phi_{1}(1,1)\right], \\
& \gamma_{x y}^{0}=-\frac{1}{E_{1}}\left[2(1+v) f_{, x y}+E_{2} k_{x y}^{1}+E_{4} k_{x y}^{3}\right],
\end{aligned}
$$

where the stress function $f(x, y, t)$ is defined as:

$$
N_{x}=f_{, y y}, N_{y}=f_{, x x}, N_{x y}=-f_{, x y} .
$$

Replacing Eq. (17) into Eqs. (13a) and (13b) gives:

$$
\begin{aligned}
& \frac{\partial^{2} u}{\partial t^{2}}=-\frac{\overline{I_{2}}}{\overline{I_{1}}} \frac{\partial^{2} \phi_{x}}{\partial t^{2}}+\frac{\overline{I_{3}}}{\overline{I_{1}}} \frac{\partial^{3} \mathrm{w}}{\partial t^{2} \partial x}, \\
& \frac{\partial^{2} v}{\partial t^{2}}=-\frac{\overline{I_{2}^{*}}}{\overline{I_{1}^{*}}} \frac{\partial^{2} \phi_{y}}{\partial t^{2}}+\frac{\overline{I_{3}^{*}}}{\overline{I_{1}^{*}}} \frac{\partial^{3} \mathrm{w}}{\partial t^{2} \partial y} .
\end{aligned}
$$

Substituting Eqs. (18a) and (18b) into Eqs. (13c-13e) yields:

$$
\begin{gathered}
Q_{x, x}+Q_{y, y}-3 c_{1}\left(K_{x, x}+K_{y, y}\right)+c_{1}\left(P_{x, x x}+2 P_{x y, x y}+P_{y, y y}\right)+f_{, y y} \mathrm{w}_{, x x}-2 f_{, x y} \mathrm{w}_{, x y}+f_{, x x} \mathrm{w}_{, y y} \\
+p-k_{1} \mathrm{w}+k_{2} \nabla^{2} \mathrm{w}=I_{1} \frac{\partial^{2} w}{\partial t^{2}}+2 \varepsilon I_{1} \frac{\partial \mathrm{w}}{\partial t}+\overline{\overline{I_{5}}}\left(\frac{\partial^{3} \phi_{x}}{\partial t^{2} \partial x}+\frac{\partial^{3} \phi_{y}}{\partial t^{2} \partial y}\right)+\overline{\overline{I_{7}}}\left(\frac{\partial^{4} \mathrm{w}}{\partial t^{2} \partial x^{2}}+\frac{\partial^{4} \mathrm{w}}{\partial t^{2} \partial y^{2}}\right), \\
M_{x, x}+M_{x y, y}-Q_{x}+3 c_{1} K_{x}-c_{1}\left(P_{x, x}+P_{x y, y}\right)=\overline{\overline{I_{3}}} \frac{\partial^{2} \phi_{x}}{\partial t^{2}}-\overline{\overline{I_{5}}} \frac{\partial^{3} \mathrm{w}}{\partial t^{2} \partial x}, \\
M_{x y, x}+M_{y, y}-Q_{y}+3 c_{1} K_{y}-c_{1}\left(P_{x y, x}+P_{y, y}\right)=\overline{\overline{I_{3}}} \frac{\partial^{2} \phi_{y}}{\partial t^{2}}-\overline{\overline{I_{5}}} \frac{\partial^{3} \mathrm{w}}{\partial t^{2} \partial y},
\end{gathered}
$$

in which, 


\section{ACCEPTED MANUSCRIPT}

$$
\overline{\overline{I_{3}}}=\overline{I_{4}}-\left(\overline{I_{2}}\right)^{2} / I_{1}, \overline{\overline{I_{5}}}=\overline{I_{5}}-\overline{I_{2}} \overline{I_{3}} / I_{1}, \overline{\overline{I_{7}}}=\left(\overline{I_{3}}\right)^{2} / I_{1}-c_{1}^{2} I_{7}
$$

Introducing of Eq. (16) into Eq. (11) and then into Eqs. (19) gives:

$$
\begin{aligned}
& R_{11}(w)+R_{12}\left(\phi_{x}\right)+R_{13}\left(\phi_{y}\right)+S(w, f)+p= \\
& =I_{1} \frac{\partial^{2} w}{\partial t^{2}}+2 \varepsilon I_{1} \frac{\partial \mathrm{w}}{\partial t}+\overline{\overline{I_{5}}}\left(\frac{\partial^{3} \phi_{x}}{\partial t^{2} \partial x}+\frac{\partial^{3} \phi_{y}}{\partial t^{2} \partial y}\right)+\overline{\overline{I_{7}}}\left(\frac{\partial^{4} \mathrm{w}}{\partial t^{2} \partial x^{2}}+\frac{\partial^{4} \mathrm{w}}{\partial t^{2} \partial y^{2}}\right) \\
& R_{21}(w)+R_{22}\left(\phi_{x}\right)+R_{23}\left(\phi_{y}\right)=\overline{\overline{I_{3}}} \frac{\partial^{2} \phi_{x}}{\partial t^{2}}-\overline{\overline{I_{5}}} \frac{\partial^{3} \mathrm{w}}{\partial t^{2} \partial x}, \\
& R_{31}(w)+R_{32}\left(\phi_{x}\right)+R_{33}\left(\phi_{y}\right)=\overline{\overline{I_{3}}} \frac{\partial^{2} \phi_{y}}{\partial t^{2}}-\overline{\overline{I_{5}}} \frac{\partial^{3} \mathrm{w}}{\partial t^{2} \partial y},
\end{aligned}
$$

with,

$$
\begin{aligned}
& R_{11}(w)=\left(D_{6}+k_{2}\right) \nabla^{2} \mathrm{w}-c_{1}^{2} D_{3} \nabla^{4} \mathrm{w}-k_{1} w, \\
& R_{12}\left(\phi_{x}\right)=D_{6} \phi_{x, x}+c_{1} D_{5}\left(\phi_{x, x x x}+\phi_{x, x y y}\right), \\
& R_{13}\left(\phi_{y}\right)=D_{6} \phi_{y, y}+c_{1} D_{5}\left(\phi_{y, x x y}+\phi_{y, y y y}\right), \\
& S(w, f)=f_{, y y} w_{, x x}-2 f_{, x y} \mathrm{w}_{, x y}+f_{, x x} \mathrm{w}_{, y y}, \\
& R_{21}(w)=-D_{6} \mathrm{w}_{, x}-c_{1} D_{5}\left(\mathrm{w}_{, x x x}+\mathrm{w}_{, x y y}\right), \\
& R_{22}\left(\phi_{x}\right)=D_{7} \phi_{x, x x}+D_{8} \phi_{x, y y}-D_{6} \phi_{x}, \\
& R_{23}\left(\phi_{y}\right)=D_{9} \phi_{y, x y}, \\
& R_{31}(w)=-D_{6} \mathrm{w}_{, y}-c_{1} D_{5}\left(\mathrm{w}_{, y y y}+\mathrm{w}_{, x x y}\right), \\
& R_{32}\left(\phi_{x}\right)=D_{9} \phi_{x, x y}, \\
& R_{33}\left(\phi_{y}\right)=D_{7} \phi_{y, x y}+D_{8} \phi_{y, x x}-D_{6} \phi_{y}, \\
& D_{1}=\frac{E_{1} E_{3}-E_{2}^{2}}{E_{1}\left(1-v^{2}\right)}, D_{2}=\frac{E_{1} E_{5}-E_{2} E_{4}}{E_{1}\left(1-v^{2}\right)}, D_{3}=\frac{E_{1} E_{7}-E_{4}^{2}}{E_{1}\left(1-v^{2}\right)}, \\
& D_{4}=D_{1}-c_{1} D_{2}, D_{5}=D_{2}-c_{1} D_{3}, D_{6}=\frac{1}{2(1+v)}\left(E_{1}-6 c_{1} E_{3}+9 c_{1}^{2} E_{5}\right), \\
& D_{7}=D_{4}-c_{1} D_{5}, D_{8}=\frac{(1-v)}{2}\left(D_{4}-c_{1} D_{5}\right), D_{9}=\frac{(1+v)}{2}\left(D_{4}-c_{1} D_{5}\right) .
\end{aligned}
$$




\section{ACCEPTED MANUSCRIPT}

For an imperfect FGM plate, Eqs. (21) may be transformed to the following form:

$$
\begin{aligned}
& R_{11}(w)+R_{12}\left(\phi_{x}\right)+R_{13}\left(\phi_{y}\right)+S(w, f)+S_{1}\left(w^{*}, f\right)+p= \\
& =I_{1} \frac{\partial^{2} w}{\partial t^{2}}+2 \varepsilon I_{1} \frac{\partial \mathrm{w}}{\partial t}+\overline{\overline{I_{5}}}\left(\frac{\partial^{3} \phi_{x}}{\partial t^{2} \partial x}+\frac{\partial^{3} \phi_{y}}{\partial t^{2} \partial y}\right)+\overline{\overline{I_{7}}}\left(\frac{\partial^{4} \mathrm{w}}{\partial t^{2} \partial x^{2}}+\frac{\partial^{4} \mathrm{w}}{\partial t^{2} \partial y^{2}}\right), \\
& R_{21}(w)+R_{22}\left(\phi_{x}\right)+R_{23}\left(\phi_{y}\right)+R_{21}^{*}\left(w^{*}\right)=\overline{\overline{I_{3}}} \frac{\partial^{2} \phi_{x}}{\partial t^{2}}-\overline{\overline{I_{5}}} \frac{\partial^{3} \mathrm{w}}{\partial t^{2} \partial x}, \\
& R_{31}(w)+R_{32}\left(\phi_{x}\right)+R_{33}\left(\phi_{y}\right)+R_{31}^{*}\left(w^{*}\right)=\overline{\overline{I_{3}}} \frac{\partial^{2} \phi_{y}}{\partial t^{2}}-\overline{\overline{I_{5}}} \frac{\partial^{3} \mathrm{w}}{\partial t^{2} \partial y},
\end{aligned}
$$

in which the imperfection function $w^{*}(x, y)$ represents an initial small deviation of the plate surface from a perfect configuration, and

$$
\begin{aligned}
& R_{21}^{*}\left(w^{*}\right)=-D_{6} \mathrm{w}_{, x}, \\
& R_{31}^{*}\left(w^{*}\right)=-D_{6} \mathrm{w}_{, y}, \\
& S_{1}\left(w^{*}, f\right)=D_{6}\left(w^{*}{ }_{, x x}+\mathrm{w}^{*}{ }_{, y y}\right)+f_{, y y} w^{*}{ }_{, x x}-2 f_{, x y} \mathrm{w}_{, x y}{ }_{, x}+f_{, x x} \mathrm{w}^{*}{ }_{, y y} .
\end{aligned}
$$

The geometrical compatibility equation for an imperfect FGM plate may be derived as [11]:

$$
\varepsilon_{x, y y}^{0}+\varepsilon_{y, x x}^{0}-\gamma_{x y, x y}^{0}=w_{, x y}^{2}-w_{, x x} w_{, y y}+2 w_{, x y} w_{, x y}^{*}-w_{, x x} w_{, y y}^{*}-w_{, y y} w_{, x x}^{*} .
$$

Inserting Eqs. (16) into Eq. (25), the compatibility equation of an imperfect FGM plate is given as:

$$
\nabla^{4} f-E_{1}\left(w_{, x y}^{2}-w_{, x x} w_{, y y}+2 w_{, x y} w_{, x y}^{*}-w_{, x x} w_{, y y}^{*}-w_{, y y} w_{, x x}^{*}\right)=0
$$

The nonlinear Eqs. (23) and (26) are used to investigate the nonlinear vibration and dynamic 


\section{ACCEPTED MANUSCRIPT}

stability of imperfect FGM thick plates using the higher-order shear deformation theory.

\section{Solution procedure}

\subsection{Boundary conditions}

Suppose that two cases of boundary conditions will be considered, as shown below:

Case 1: Four edges of the plate are simply supported. Assume an imperfect FGM plate with immovable edges is under blast load $p$. Thus, the boundary conditions are:

$$
\begin{aligned}
& \mathrm{W}=N_{x y}=\phi_{y}=M_{x}=0, N_{x}=N_{x 0} \text { at } x=0, a \\
& \mathrm{~W}=N_{x y}=\phi_{x}=M_{y}=0, N_{y}=N_{y 0} \text { at } y=0, b
\end{aligned}
$$

where $N_{x 0}, N_{y 0}$ are fictitious compressive edge loads at immovable edges.

Case 2: Two edges $x=0$ and $x=a$ are simply supported, and the remaining two edges $y=0, y=b$ are clamped. The imperfect FGM plate is under blast load $p$. The boundary conditions are:

$$
\begin{aligned}
& \mathrm{W}=N_{x y}=\phi_{y}=M_{x}=0, N_{x}=N_{x 0} \text { at } x=0, a \\
& \mathrm{~W}=\frac{\partial \mathrm{w}}{\partial y}=N_{x y}=\phi_{x}=\phi_{y}=N_{y}=0 \text { at } y=0, b
\end{aligned}
$$

\subsection{FGM plate with simply supported four edges}

The approximate solution satisfying the abovementioned boundary conditions in Eq. (27) may be found in the following form:

$$
\left[\begin{array}{c}
\mathrm{w}(x, y, t) \\
\phi_{x}(x, y, t) \\
\phi_{y}(x, y, t)
\end{array}\right]=\left[\begin{array}{c}
\mathrm{W}(t) \sin \lambda_{m} x \sin \delta_{n} y \\
\Phi_{x}(t) \cos \lambda_{m} x \sin \delta_{n} y \\
\Phi_{y}(t) \sin \lambda_{m} x \cos \delta_{n} y
\end{array}\right],
$$




\section{ACCEPTED MANUSCRIPT}

where $\lambda_{m}=\frac{m \pi}{a}, \delta_{n}=\frac{n \pi}{b}$, and $m, n=1,2, \ldots$ are the natural numbers of half-waves in the corresponding direction $x, y . W(t), \Phi_{x}, \Phi_{y}$ are the time-dependent amplitudes.

With regard to the initial imperfection $w^{*}$, we introduce an assumption that it has a form like the plate deflection, i.e.:

$$
\mathrm{w}^{*}(x, y)=\mathrm{W}_{0} \sin \lambda_{m} x \sin \delta_{n} y,
$$

in which $\mathrm{W}_{0}$ is the known initial amplitude.

Introducing Eqs. (29) and (30) into the compatibility Equation (26), and solving the obtained equation for the unknown $f$, leads to:

$$
f=A_{1} \cos 2 \lambda_{m} x+A_{2} \cos 2 \delta_{n} y+\frac{1}{2} N_{x 0} y^{2}+\frac{1}{2} N_{y 0} x^{2}
$$

with,

$$
A_{1}=\frac{E_{1} \delta_{n}^{2}}{32 \lambda_{m}^{2}} W\left(W+2 W_{0}\right), A_{2}=\frac{E_{1} \lambda_{m}^{2}}{32 \delta_{n}^{2}} W\left(W+2 W_{0}\right) .
$$

Replacing Eqs. (29)-(31) into Eqs. (23), and then applying the Galerkin method to the resulting equations yields:

$$
\begin{aligned}
& r_{11} \mathrm{~W}+r_{12} \Phi_{x}+r_{13} \Phi_{y}+\left[s_{1}-\left(N_{x 0} \lambda_{m}^{2}+N_{y 0} \delta_{n}^{2}\right)\right]\left(W+W_{0}\right) \\
& +s_{2} W\left(W+W_{0}\right)\left(W+2 W_{0}\right)+s_{3} p=I_{0} \frac{\partial^{2} \mathrm{~W}}{\partial t^{2}}+2 \varepsilon I_{1} \frac{\partial \mathrm{W}}{\partial t} \\
& -\overline{\overline{I_{5}}} \lambda_{m} \frac{\partial^{2} \Phi_{x}}{\partial t^{2}}-\overline{\overline{I_{5}}} \delta_{n} \frac{\partial^{2} \Phi_{y}}{\partial t^{2}}
\end{aligned}
$$




\section{ACCEPTED MANUSCRIPT}

$$
\begin{aligned}
& r_{21} \Phi_{x}+r_{22} \Phi_{y}+s_{4}\left(W+W_{0}\right)=\overline{\overline{I_{3}}} \frac{\partial^{2} \Phi_{x}}{\partial t^{2}}-\overline{\overline{I_{5}}} \lambda_{m} \frac{\partial^{2} \mathrm{~W}}{\partial t^{2}}, \\
& r_{31} \Phi_{x}+r_{32} \Phi_{y}+s_{5}\left(W+W_{0}\right)=\overline{\overline{I_{3}}} \frac{\partial^{2} \Phi_{y}}{\partial t^{2}}-\overline{\overline{I_{5}}} \delta_{n} \frac{\partial^{2} \mathrm{~W}}{\partial t^{2}},
\end{aligned}
$$

in which the detail of the coefficients $I_{0}, r_{1 i}(i=\overline{1,3}), r_{j k}(j=\overline{2,3}, k=\overline{1,2}), s_{m}(m=\overline{1,5})$ may be found in Appendix A.

The plate is subjected to blast load $p$ (Pascals) and simultaneously exposed to temperature environments. The in-plane condition on immovability at all edges, i.e. $u=0$ at $x=0, a$ and $v=0$ at $y=0, b$, is fulfilled in an average sense as $[6,11]$ :

$$
\int_{0}^{b} \int_{0}^{a} \frac{\partial u}{\partial x} d x d y=0, \int_{0}^{a} \int_{0}^{b} \frac{\partial v}{\partial y} d y d x=0
$$

From Eqs. (8) and (16), one can obtain the following expressions in which the imperfection has been included:

$$
\begin{aligned}
& \frac{\partial u}{\partial x}=\frac{1}{E_{1}}\left(f_{, y y}-v f_{, x x}\right)-\frac{E_{2}}{E_{1}} \phi_{x, x}+\frac{c_{1} E_{4}}{E_{1}}\left(\phi_{x, x}+w_{, x x}\right)-\frac{1}{2} w_{, x}^{2}-w_{, x} w_{, x}^{*}+\frac{\Phi_{1}}{E_{1}}, \\
& \frac{\partial v}{\partial y}=\frac{1}{E_{1}}\left(f_{, x x}-v f_{, y y}\right)-\frac{E_{2}}{E_{1}} \phi_{y, y}+\frac{c_{1} E_{4}}{E_{1}}\left(\phi_{y, y}+w_{, y y}\right)-\frac{1}{2} w_{, y}^{2}-w_{, y} w_{, y}^{*}+\frac{\Phi_{1}}{E_{1}}
\end{aligned}
$$

Substitution of Eqs. (29)-(31) into Eqs. (35) and (36) then result in Eqs. (34), giving fictitious edge compressive loads as: 


\section{ACCEPTED MANUSCRIPT}

$$
\begin{aligned}
& N_{x 0}=-\frac{\Phi_{1}}{1-v}-\frac{4}{m n \pi^{2}\left(1-v^{2}\right)}\left[\left(E_{2}-c_{1} E_{4}\right)\left(\lambda_{m} \Phi_{x}+v \delta_{n} \Phi_{y}\right)-c_{1} E_{4}\left(\lambda_{m}^{2}+v \delta_{n}^{2}\right) W\right] \\
& +\frac{E_{1}}{8\left(1-v^{2}\right)}\left(\lambda_{m}^{2}+v \delta_{n}^{2}\right) W\left(W+2 W_{0}\right), \\
& N_{y 0}=-\frac{\Phi_{1}}{1-v}-\frac{4}{m n \pi^{2}\left(1-v^{2}\right)}\left[\left(E_{2}-c_{1} E_{4}\right)\left(v \lambda_{m} \Phi_{x}+\delta_{n} \Phi_{y}\right)-c_{1} E_{4}\left(v \lambda_{m}^{2}+\delta_{n}^{2}\right) W\right] \\
& +\frac{E_{1}}{8\left(1-v^{2}\right)}\left(v \lambda_{m}^{2}+\delta_{n}^{2}\right) W\left(W+2 W_{0}\right),
\end{aligned}
$$

in which the thermal parameter is obtained from Eqs. (12) as:

$$
\Phi_{1}=L h \Delta T, L=E_{m} \alpha_{m}+\frac{E_{m} \alpha_{c m}+E_{c m} \alpha_{m}}{N+1}+\frac{E_{c m} \alpha_{c m}}{2 N+1} .
$$

Replacing Eqs. (37), and (38) into the equations of motion (Eq. (33)), we have:

$$
\begin{aligned}
& r_{11} \mathrm{~W}+r_{12} \Phi_{x}+r_{13} \Phi_{y}+\left[s_{1}+\frac{\Phi_{1}}{1-v}\left(\lambda_{m}^{2}+\delta_{n}^{2}\right)\right]\left(W+W_{0}\right) \\
& +\overline{s_{2}} W\left(W+W_{0}\right)\left(W+2 W_{0}\right)+h_{1} \Phi_{x}\left(W+W_{0}\right) \\
& +h_{2} \Phi_{y}\left(W+W_{0}\right)+h_{3} W\left(W+W_{0}\right)+s_{3} p=I_{0} \frac{\partial^{2} \mathrm{~W}}{\partial t^{2}} \\
& +2 \varepsilon I_{1} \frac{\partial \mathrm{W}}{\partial t}-\overline{\bar{I}_{5}} \lambda_{m} \frac{\partial^{2} \Phi_{x}}{\partial t^{2}}-\overline{\overline{I_{5}}} \delta_{n} \frac{\partial^{2} \Phi_{y}}{\partial t^{2}}, \\
& r_{21} \Phi_{x}+r_{22} \Phi_{y}+s_{4}\left(W+W_{0}\right)=\overline{\overline{I_{3}}} \frac{\partial^{2} \Phi_{x}}{\partial t^{2}}-\overline{\overline{I_{5}}} \lambda_{m} \frac{\partial^{2} \mathrm{~W}}{\partial t^{2}} \\
& r_{31} \Phi_{x}+r_{32} \Phi_{y}+s_{5}\left(W+W_{0}\right)=\overline{\overline{I_{3}}} \frac{\partial^{2} \Phi_{y}}{\partial t^{2}}-\overline{\overline{I_{5}}} \delta_{n} \frac{\partial^{2} \mathrm{~W}}{\partial t^{2}}
\end{aligned}
$$

with the specific expressions of the coefficients $h_{i}(i=\overline{1,3}), \overline{s_{2}}$ given in Appendix A. 


\section{ACCEPTED MANUSCRIPT}

By using the fourth-order Runge-Kutta method, the nonlinear dynamic responses of the simply supported FGM plates are investigated by solving the system of Eqs. (40), combined with the initial conditions.

Taking linear parts of Eqs. (40) and making $p=0$, the natural frequencies of the perfect simply supported plate is the smallest value of three frequencies, $\omega$, which can be determined by solving the following determinant:

$$
\left|\begin{array}{ccc}
r_{11}+s_{1}+\frac{\Phi_{1}}{1-v}\left(\lambda_{m}^{2}+\delta_{n}^{2}\right)+I_{0} \omega^{2} & r_{12}-\overline{\overline{I_{5}}} \lambda_{m} \omega^{2} & r_{13}-\overline{\overline{I_{5}}} \delta_{n} \omega^{2} \\
s_{4}-\overline{\overline{I_{5}}} \lambda_{m} \omega^{2} & r_{21}+\overline{\overline{I_{3}}} \omega^{2} & r_{22} \\
s_{5}-\overline{\overline{I_{5}}} \delta_{n} \omega^{2} & r_{31} & r_{32}+\overline{\overline{I_{3}}} \omega^{2}
\end{array}\right|=0 .
$$

\subsection{FGM plate with two simply supported edges and two clamped edges}

The approximate solutions of $w, \phi_{x}$ and $\phi_{y}$ satisfying the boundary conditions from Eq. (28) are assumed to be:

$$
\left[\begin{array}{l}
\mathrm{w}(x, y, t) \\
\phi_{x}(x, y, t) \\
\phi_{y}(x, y, t)
\end{array}\right]=\left[\begin{array}{c}
\mathrm{W}(t) \sin \lambda_{m} x\left(1-\cos 2 \delta_{n} y\right) \\
\Phi_{x}(t) \cos \lambda_{m} x \sin \delta_{n} y \\
\Phi_{y}(t) \sin \lambda_{m} x \sin \delta_{n} y
\end{array}\right] .
$$

The initial imperfection $w^{*}$ is assumed to be in the form:

$$
\mathrm{w}^{*}(x, y)=\mathrm{W}_{0} \sin \lambda_{m} x\left(1-\cos 2 \delta_{n} y\right) .
$$

Substituting Eqs. (42) and (43) into the compatibility Equation (26), we define the stress function as: 


\section{ACCEPTED MANUSCRIPT}

$f=B_{1} \cos 2 \lambda_{m} x+B_{2} \cos 2 \delta_{n} y+B_{3} \cos 4 \delta_{n} y+B_{4} \cos 2 \lambda_{m} x \cos 2 \delta_{n} y+\frac{1}{2} N_{x 0} y^{2}$

)

with,

$$
\begin{aligned}
& B_{1}=\frac{E_{1} \delta_{n}^{2}}{8 \lambda_{m}^{2}} W\left(W+2 W_{0}\right), B_{2}=\frac{E_{1} \lambda_{m}^{2}}{8 \delta_{n}^{2}} W\left(W+2 W_{0}\right) \\
& B_{3}=\frac{-E_{1} \lambda_{m}^{2}}{128 \delta_{n}^{2}} W\left(W+2 W_{0}\right), B_{4}=\frac{-E_{1} \lambda_{m}^{2} \delta_{n}^{2}}{8\left(\lambda_{m}^{2}+\delta_{n}^{2}\right)^{2}} W\left(W+2 W_{0}\right) .
\end{aligned}
$$

Subsequently, substitution of Eqs. (42)-(44) into Eq. (23), and applying the Galerkin procedure for the resulting equation yields:

$$
\begin{gathered}
l_{11} W+l_{12} \Phi_{x}+\left(n_{1}-N_{x 0} \lambda_{m}^{2}\right)\left(W+W_{0}\right)+n_{2} W\left(W+W_{0}\right)\left(W+2 W_{0}\right) \\
+n_{3} p=\overline{I_{0}} \frac{\partial^{2} W}{\partial t^{2}}+2 \varepsilon I_{1} \frac{\partial W}{\partial t}-\overline{\overline{I_{5}}} \frac{16 \lambda_{m}}{9 b \delta_{n}} \frac{\partial^{2} \Phi_{x}}{\partial t^{2}} \\
l_{21} \mathrm{~W}+l_{22} \Phi_{x}+n_{4}\left(W+W_{0}\right)=\overline{\overline{I_{3}}} \frac{\partial^{2} \Phi_{x}}{\partial t^{2}}-\overline{\overline{I_{5}}} \frac{16 \lambda_{m}}{3 b \delta_{n}} \frac{\partial^{2} W}{\partial t^{2}} \\
l_{31} \Phi_{y}=\overline{I_{3}} \frac{\partial^{2} \Phi_{y}}{\partial t^{2}},
\end{gathered}
$$

in which the detail of coefficients $l_{i j}(i, j=\overline{1,2}), l_{31}, n_{m}(m=\overline{1,4})$ may be found in Appendix B.

The in-plane condition on immovability at $y=0, b$, i.e. $v=0$ at $y=0, b$, is fulfilled in an average sense as:

$$
\int_{0}^{a} \int_{0}^{b} \frac{\partial v}{\partial y} d y d x=0
$$




\section{ACCEPTED MANUSCRIPT}

Putting Eqs. (42)-(44) into Eq. (36), then substituting the obtained result into Eq. (47) leads

to:

$$
N_{x 0}=\frac{\Phi_{1}}{v}+\frac{E_{1} \delta_{n}^{2}}{2 v} W\left(W+2 W_{0}\right) .
$$

Substituting Eq. (48) into Eqs. (46), the system of motion of Eqs. (46) can be rewritten as follows:

$$
\begin{gathered}
l_{11} W+l_{12} \Phi_{x}+\left(n_{1}-\frac{\Phi_{1}}{v} \lambda_{m}^{2}\right)\left(W+W_{0}\right)+\overline{n_{2}} W\left(W+W_{0}\right)\left(W+2 W_{0}\right) \\
+n_{3} p=\overline{I_{0}} \frac{\partial^{2} W}{\partial t^{2}}+2 \varepsilon I_{1} \frac{\partial W}{\partial t}-\overline{\overline{I_{5}}} \frac{16 \lambda_{m}}{9 b \delta_{n}} \frac{\partial^{2} \Phi_{x}}{\partial t^{2}} \\
l_{21} \mathrm{~W}+l_{22} \Phi_{x}+n_{4}\left(W+W_{0}\right)=\overline{\bar{I}_{3}} \frac{\partial^{2} \Phi_{x}}{\partial t^{2}}-\overline{I_{5}} \frac{16 \lambda_{m}}{3 b \delta_{n}} \frac{\partial^{2} W}{\partial t^{2}} \\
l_{31} \Phi_{y}=\overline{\bar{I}_{3}} \frac{\partial^{2} \Phi_{y}}{\partial t^{2}}
\end{gathered}
$$

where the coefficient $\overline{n_{2}}$ is described in detail in Appendix B.

This is the system of equations used to investigate the dynamic characteristics of imperfect FGM plates with two edges simply supported and two edges clamped by using the fourth-order Runge-Kutta method.

\section{Numerical results and discussion}

The effective material properties mentioned in Eq. (5) are listed in Table $1[6,7,11]$. The Poisson's ratio is $v=0.3$. The initial conditions are chosen as follows: 


\section{ACCEPTED MANUSCRIPT}

$$
W(0)=0, \frac{d W}{d t}(0)=0, \Phi_{x}(0)=0, \frac{d \Phi_{x}}{d t}(0)=0, \Phi_{y}(0)=0, \frac{d \Phi_{y}}{d t}(0)=0 .
$$

\subsection{Comparative study}

In order to verify the accuracy of the proposed formulation, Figure 2 shows the comparison of nonlinear dynamic responses of the FGM plate $(b / a=1, b / h=20)$ without elastic foundations subjected to blast load in this paper based on the analytical approach and the results according to finite element method using Abaqus software. As can be seen, a good agreement is obtained in this comparison.

\subsection{Natural frequency}

Table 2 presents the natural frequency of the simply supported FGM plate with various temperature increments, $\Delta T$, and coefficients $k_{1}(G P a / m), k_{2}(G P a . m)$ of the Winkler and Pasternak foundations. From the results in this table, it can be seen that the natural frequency of the plate increases when the temperature increment $\Delta T$ decreases and coefficients $k_{1}, k_{2}$ increase. The effect of volume fraction index, $N$, on the natural frequency of the FGM plate is also shown in Table 2. Obviously, an increase of the volume fraction index leads to a decrease of the natural frequency of the FGM plate.

\subsection{Effects of temperature increment}

Figures 3 and 4 show the effects of temperature increment, $\Delta T$, on the nonlinear dynamic response of the simply supported FGM plate and the FGM plate with two edges simply 


\section{ACCEPTED MANUSCRIPT}

supported and two edges clamped under conditions: $b / a=1, b / h=20, m=n=1$, $N=1, k_{1}=0.3 \mathrm{GPa} / \mathrm{m}$, and $k_{2}=0.02 \mathrm{GPa} . \mathrm{m}$. As expected, an increase in temperature increment leads to a rise of the absolute value of the FGM plate amplitude. The results also show that the absolute value of the simply supported FGM plate is higher than the FGM plate with two edges simply supported and two edges clamped.

\subsection{Effects of elastic foundations}

Figures 5-8 present the effects of coefficients $k_{1}, k_{2}$ of the Winkler and Pasternak foundations on the nonlinear dynamic response of the simply supported FGM plate and the FGM plate with two edges simply supported and two edges clamped under blast and thermal load. It is easy to see that the absolute value of FGM plate amplitude fluctuation decreases when these modules increase. In addition, compared to the case corresponding to the coefficient $k_{1}$ of the Winkler model, the Pasternak type elastic foundation with coefficient $k_{2}$ has a stronger effect.

\subsection{Effects of volume fraction index}

Figures 9 and 10 show the effect of volume fraction index, $N$, on the nonlinear dynamic response of the simply supported FGM plate and the FGM plate with two edges simply supported and two edges clamped, with: $\quad b / a=1, b / h=20, \Delta T=350 \mathrm{~K}$ $k_{1}=0.3 \mathrm{GPa} / \mathrm{m}, k_{2}=0.02 \mathrm{GPa} . \mathrm{m}$, respectively. As can be seen, the absolute value of nonlinear dynamic response amplitude of the FGM plate decreases when the power-law index 


\section{ACCEPTED MANUSCRIPT}

$N$ decreases. This is reasonable because when $N$ is decreased, the ceramic volume fraction is increased; however, the elastic module of the ceramic is higher than metal $\left(E_{c}>E_{m}\right)$.

\subsection{Effects of initial imperfection}

The influences of initial imperfection with amplitude $W_{0}$ on the nonlinear dynamic response of the simply supported FGM plate, and the FGM plate with two edges simply supported and two edges clamped are shown in Figures 11 and 12, respectively. Three values of $W_{0}: 0,10^{-4} \mathrm{~m}$ and $3 \times 10^{-4} \mathrm{~m}$ are used. As can be observed, the reduction of the amplitude of the initial imperfection leads to the decrease of the absolute value of the FGM plate amplitude fluctuation.

\subsection{Effects of geometrical parameters}

Figures 13-16 indicate the effects of geometrical parameters on the nonlinear dynamic response of the simply supported FGM plate and the FGM plate with two edges simply supported and two edges clamped under blast and thermal loads. Specifically, Figures 13 and 14 consider the influences of ratio $b / a$ on the nonlinear dynamic response of the simply supported FGM plate, and two edges supported and two edges clamped FGM plate respectively. The nonlinear dynamic responses of the FGM plates with various values of ratio $b / h$ are illustrated in Figures 15 and 16. Obviously, the absolute value of the FGM plate amplitude increases when increasing the ratios $b / a$ and $b / h$. 


\section{ACCEPTED MANUSCRIPT}

\section{Conclusions}

This paper presents an analytical solution to investigate the nonlinear vibration and dynamic response of imperfect FGM thick plates resting on elastic foundations under blast and thermal loads using Reddy's higher-order shear deformation plate theory. Material properties are assumed to be temperature-dependent and graded in the thickness direction according to a simple power-law distribution in terms of the volume fractions of the constituents. By using the Galerkin method the equation system of motion to determine dynamic response is found. The numerical results are investigated by the Runge-Kutta procedure.

Some special conclusions are obtained for the FGM plate subjected to blast and thermal loads:

- $\quad$ The temperature strongly influences the nonlinear dynamic response and vibration of FGM plates.

The elastic foundations significantly enhance the load-carrying capacity of the FGM plates. In addition, the stiffness $k_{2}$ of the shear layer of the Pasternak foundation model has a more pronounced effect on the nonlinear dynamic stability of FGM plates than the modulus $k_{1}$ of the Winkler model.

- The geometrical dimensions affect considerably on the nonlinear dynamic response of the FGM plates.

The initial imperfection increases the dynamic fluctuation amplitude of the FGM plates.

\section{Funding}




\section{ACCEPTED MANUSCRIPT}

This work was supported by the Grant in Mechanics of the National Foundation for Science and

Technology Development of Vietnam - NAFOSTED, code 107.02-2013.06. The authors are grateful for this support.

\section{Appendix A}

$$
\begin{aligned}
& I_{o}=\left(I_{1}-I_{7} \frac{m^{2} \pi^{2}}{a^{2}}-I_{7}^{*} \frac{n^{2} \pi^{2}}{b^{2}}\right) \\
& r_{11}=-\left[c_{1}^{2} D_{3}\left(\frac{m^{2} \pi^{2}}{a^{2}}+\frac{n^{2} \pi^{2}}{b^{2}}\right)^{2}+k_{1}+k_{2}\left(\frac{m^{2} \pi^{2}}{a^{2}}+\frac{n^{2} \pi^{2}}{b^{2}}\right)\right], \\
& r_{12}=-D_{6} \frac{m \pi}{a}+c_{1} D_{5} \frac{m^{3} \pi^{3}}{a^{3}}+c_{1} D_{5} \frac{m \pi}{a} \frac{n^{2} \pi^{2}}{b^{2}}, \\
& r_{13}=-D_{6} \frac{n \pi}{b}+c_{1} D_{5} \frac{n^{3} \pi^{3}}{b^{3}}+c_{1} D_{5} \frac{n \pi}{b} \frac{m^{2} \pi^{2}}{a^{2}}, \\
& s_{1}=-D_{6}\left(\frac{m^{2} \pi^{2}}{a^{2}}+\frac{n^{2} \pi^{2}}{b^{2}}\right), s_{2}=-\frac{E_{1}}{16}\left(\frac{m^{4} \pi^{4}}{a^{4}}+\frac{n^{4} \pi^{4}}{b^{4}}\right), s_{3}=\frac{16}{m n \pi^{2}}, \\
& r_{21}=-D_{6}-D_{7} \frac{m^{2} \pi^{2}}{a^{2}}-D_{8} \frac{n^{2} \pi^{2}}{b^{2}}, r_{22}=-D_{9} \frac{m n \pi^{2}}{a b}, s_{4}=-D_{6} \frac{m \pi}{a}+c_{1} D_{5} \frac{m^{3} \pi^{3}}{a^{3}}+c_{1} D_{5} \frac{m n^{2} \pi^{3}}{a b^{2}} \\
& r_{31}=-D_{9} \frac{m n \pi^{2}}{a b}, r_{32}=-D_{6}-D_{7} \frac{n^{2} \pi^{2}}{b^{2}}-D_{8} \frac{m^{2} \pi^{2}}{a^{2}}, s_{5}=-D_{6} \frac{n \pi}{b}+c_{1} D_{5} \frac{n^{3} \pi^{3}}{b^{3}}+c_{1} D_{5} \frac{m^{2} n \pi^{3}}{a^{2} b} \\
& h_{1}=\frac{4 \lambda_{m}}{m n \pi^{2}\left(1-v^{2}\right)}\left(E_{2}-c_{1} E_{4}\right)\left(\lambda_{m}^{2}+v \delta_{n}^{2}\right), h_{2}=\frac{4 \delta_{n}}{m n \pi^{2}\left(1-v^{2}\right)}\left(E_{2}-c_{1} E_{4}\right)\left(v \lambda_{m}^{2}+\delta_{n}^{2}\right), \\
& \left.h_{4}=-\lambda_{m}^{4}+2 v \delta_{n}^{2} \lambda_{m}^{2}+\delta_{n}^{4}\right), s_{2}=s_{2}-\frac{E_{1}}{8\left(1-v^{2}\right)}\left(\lambda_{m}^{4}+2 v \delta_{n}^{2} \lambda_{m}^{2}+\delta_{n}^{4}\right)
\end{aligned}
$$




\section{ACCEPTED MANUSCRIPT}

\section{Appendix B}

$$
\begin{aligned}
& \overline{I_{0}}=I_{1}-I_{7} \frac{m^{2} \pi^{2}}{a^{2}}-\frac{4}{3} I_{7} \frac{n^{2} \pi^{2}}{b^{2}}, \\
& l_{11}=-\left[k_{1}+k_{2}\left(\frac{m^{2} \pi^{2}}{a^{2}}+\frac{4}{3} \frac{n^{2} \pi^{2}}{b^{2}}\right)+c_{1}^{2} D_{3} \frac{m^{4} \pi^{4}}{a^{4}}+\frac{16}{3} c_{1}^{2} D_{3} \frac{n^{4} \pi^{4}}{b^{4}}+\frac{8}{3} c_{1}^{2} D_{3} \frac{m^{2} n^{2} \pi^{4}}{a^{2} b^{2}}\right], \\
& l_{12}=-\frac{16 D_{6} m b}{9 b a n}+\frac{16 c_{1} D_{5} m^{3} \pi^{2}}{9 a^{3} n}+\frac{16 c_{1} D_{5} m n \pi^{2}}{9 a b^{2}}, \\
& n_{1}=-D_{6} \frac{m^{2} \pi^{2}}{a^{2}}-\frac{4}{3} D_{6} \frac{n^{2} \pi^{2}}{b^{2}}, n_{2}=-\frac{17 E_{1} m^{4} \pi^{4}}{3 a^{4}}-\frac{E_{1} m^{4} n^{4} \pi^{8}}{6\left(m^{2} \pi^{2} b^{2}+n^{2} \pi^{2} a^{2}\right)^{2}}-\frac{E_{1} n^{4} \pi^{4}}{3 b^{4}}, n_{3}=\frac{8}{3 m \pi}, \\
& l_{21}=\frac{16 c_{1} D_{5} m^{3} \pi^{2}}{3 a^{3} n}+\frac{16 c_{1} D_{5} m n \pi^{2}}{3 a b^{2}}, l_{22}=-D_{6}-D_{7} \frac{m^{2} \pi^{2}}{a^{2}}-D_{8} \frac{n^{2} \pi^{2}}{b^{2}}, \\
& n_{4}=-\frac{16 D_{6} m}{3 b a n}, l_{31}=-D_{6}-D_{7} \frac{n^{2} \pi^{2}}{b^{2}}-D_{8} \frac{m^{2} \pi^{2}}{a^{2}}, \overline{n_{2}}=n_{2}+\frac{E_{1} m^{2} n^{2} \pi^{4}}{2 v a^{2} b^{2}} .
\end{aligned}
$$




\section{ACCEPTED MANUSCRIPT}

\section{References}

[1] K. Asemi, M. Salehi, and M. Akhlaghi, Three dimensional graded finite element elasticity shear buckling analysis of FGM annular sector plates, Aero. Sci. Tech., vol. 43, pp. 1-13, 2015.

[2] M.Z. Nejad, M. Jabbari, and M. Ghannad, Elastic analysis of FGM rotating thick truncated conical shells with axially-varying properties under non-uniform pressure loading, Compos. Struct., vol. 122, pp. 561-569, 2015.

[3] L. Dozio, Exact free vibration analysis of Lévy FGM plates with higher-order shear and normal deformation theories, Compos. Struct., vol. 111, pp. 415-425, 2014.

[4] N.D. Duc, Nonlinear dynamic response of imperfect eccentrically stiffened FGM double curved shallow shells on elastic foundation, Compos. Struct., vol. 99, pp. 88-96, 2013.

[5] M.M. Alipour, and M. Shariyat, Analytical stress analysis of annular FGM sandwich plates with non-uniform shear and normal tractions, employing a zigzag-elasticity plate theory, Aero. Sci. Tech., vol. 32, no. 1, pp. 235-259, 2014.

[6] N.D. Duc, T.Q. Quan, and V.D. Luat, Nonlinear dynamic analysis and vibration of shear deformable piezoelectric FGM double curved shallow shells under damping-thermo-electromechanical loads, Compos. Struct., vol. 125, pp. 29-40, 2015.

[7] H.S. Shen, Thermal postbuckling behavior of shear deformable FGM plates with temperature-dependent properties, Int. J. Mech. Sci., vol. 49, no. 4, pp. 466-478, 2007.

[8] H.L. Dai, and Y.N. Rao, Vibration and transient response of a FGM hollow cylinder, Mech. Adv. Mater. Struct., vol. 21, no. 6, pp. 468-476, 2014. 


\section{ACCEPTED MANUSCRIPT}

[9] T.V. Loc, L.H. Anh, L. Lee, M.A. Wahab, and N.X. Hung, Vibration analysis of cracked FGM plates using higher-order shear deformation theory and extended isogeometric approach, Int. J. Mech. Sci., vol. 96-97, pp. 65-78, 2015.

[10] H. Huang, and Q. Han, Nonlinear dynamic buckling of functionally graded cylindrical shells subjected to a time-dependent axial load, Compos. Struct., vol. 92, no. 2, pp. 593-598, 2010.

[11] N.D. Duc, Nonlinear static and dynamic stability of functionally graded plates and shells, Vietnam National University Press, Hanoi 2014.

[12] Y. Shi, and M.G. Stewart, Spatial reliability analysis of explosive blast load damage to reinforced concrete columns, Struct. Safety, vol. 53, pp. 13-25, 2015.

[13] E. Gauch, J. LeBlanc, and A. Shukla, Response of preloaded thin composite panels subjected to underwater explosive loading, Comput. Struct., vol. 112-113, pp. 342-353, 2012.

[14] L.N. Ignatieva, G.A. Zverev, N.A. Adamenko, A.V. Kazurov, I.V. Sergeev, A.Y. Ustinov, I.A. Tkachenko, and V.M. Bouznik, Peculiarities of the structure of copper- and nickel-fluoropolymer composites fabricated by explosive pressing, J. Flour Chemistry, vol. 172, pp. 68-73, 2015.

[15] N. Jones, Dynamic inelastic response of strain rate sensitive ductile plates due to large impact, dynamic pressure and explosive loadings, Int. J. Impact Eng., vol. 74, pp. 3-15, 2014. 


\section{ACCEPTED MANUSCRIPT}

[16] D. Mohotti, M. Ali, N. Tuan, J. Lu, P. Mendis, and D. Ruan, Out-of-plane impact resistance of aluminium plates subjected to low velocity impacts, Mater. Des., vol. 50, pp. 413-426, 2013.

[17] A. Yehia, B.E. Din, and G.J. Dvorak, Wave propagation and dispersion in sandwich plates subjected to blast loads, Mech. Adv. Mater. Struct., vol. 14, no. 6, pp. 465475, 2007.

[18] K. Ackland, C. Anderson, N.D. Tuan, Deformation of polyurea-coated steel plates under localised blast loading, Int. J. Impact Eng., vol. 51, pp. 13-22, 2013.

[19] D.P. Gonçalves, F.C.L de Melo, A.N. Klein, and H.A. Al-Qureshi, Analysis and investigation of ballistic impact on ceramic/metal composite armour, Int. J. Mach. Tool Manu., vol. 44, no. 2-3, pp. 307-316, 2004.

[20] H.X. Luong, N.T. Chung, and N.T. Ngan, Analysis of the underground structure in the coral foundation under dynamic loading, J. Marine Sci. Tech., Vietnam, vol. 9, no. 1, pp. 10-21, 2009.

[21] C. Geretto, S.C.K. Yuen, and G.N. Nurick, An experimental study of the effects of degrees of confinement on the response of square mild steel plates subjected to blast loading, Int. J. Impact Eng., vol. 79, pp. 32-44, 2015.

[22] D. Karagiozova, G.N. Nurick, and S.C.K. Yuen, Energy absorption of aluminium alloy circular and square tubes under an axial explosive load, Thin Wall. Struct., vol. 43, no. 6, pp. 956-982, 2005. 


\section{ACCEPTED MANUSCRIPT}

[23] C. Aksoylar, A. Ömercikoğlu, Z. Mecitoğlu, and M.H. Omurtag, Nonlinear transient analysis of FGM and FML plates under blast loads by experimental and mixed FE methods, Compos. Struct., vol. 94, no. 2, pp. 731-744, 2012.

[24] T. Hause, Advanced functionally graded plate-type structures impacted by blast loading, Int. J. Impact Eng., vol. 38, no. 5, pp. 314-321, 2011.

[25] M. Übeyli, E. Balci, B. Sarikan, M.K. Öztaş, N. Camuşcu, R.O. Yildirim, and Ö. Keleş, The ballistic performance of $\mathrm{SiC}-\mathrm{AA} 7075$ functionally graded composite produced by powder metallurgy, Mater. Des., vol. 56, pp. 31-36, 2014.

[26] N. Lam, P. Mendis, P., and N. Tuan, Response spectrum solutions for blast loading, Electr. J. Struct. Eng., vol. 4, pp. 28-44, 2004.

[27] M. Bodaghi, A.R. Damanpack, M.M. Aghdam, and M. Shakeri, Non-linear active control of FG beams in thermal environments subjected to blast loads with integrated FGP sensor/actuator layers, Compos. Struct., vol. 94, no. 12, pp. 3612-3623, 2012. 


\section{ACCEPTED MANUSCRIPT}

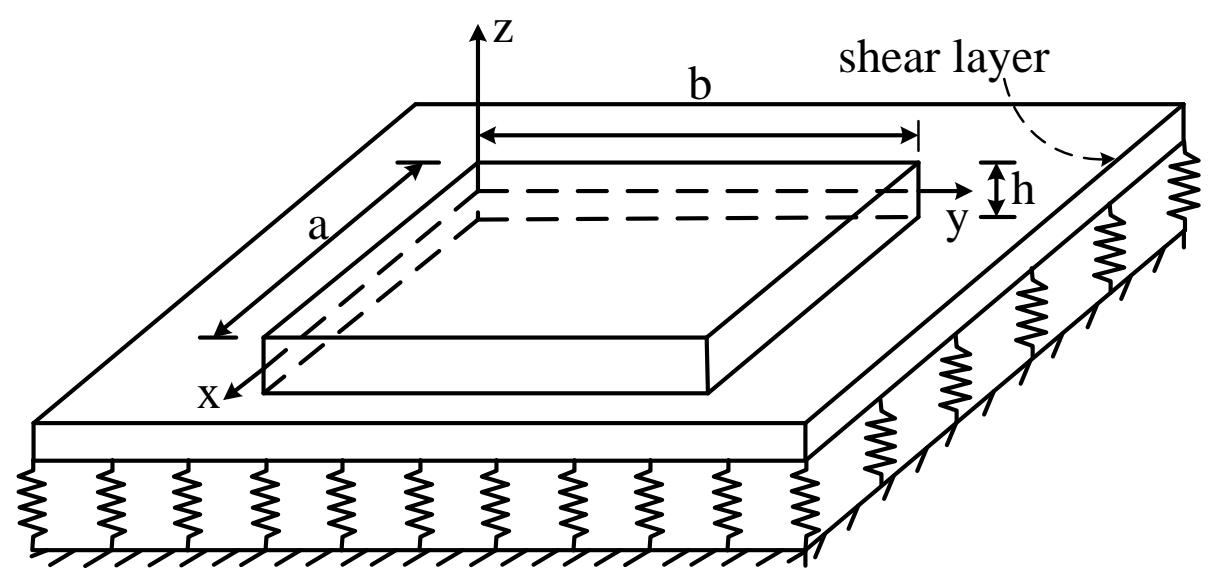

Fig. 1. Geometry and coordinate system of FGM plate on elastic foundations. 


\section{ACCEPTED MANUSCRIPT}

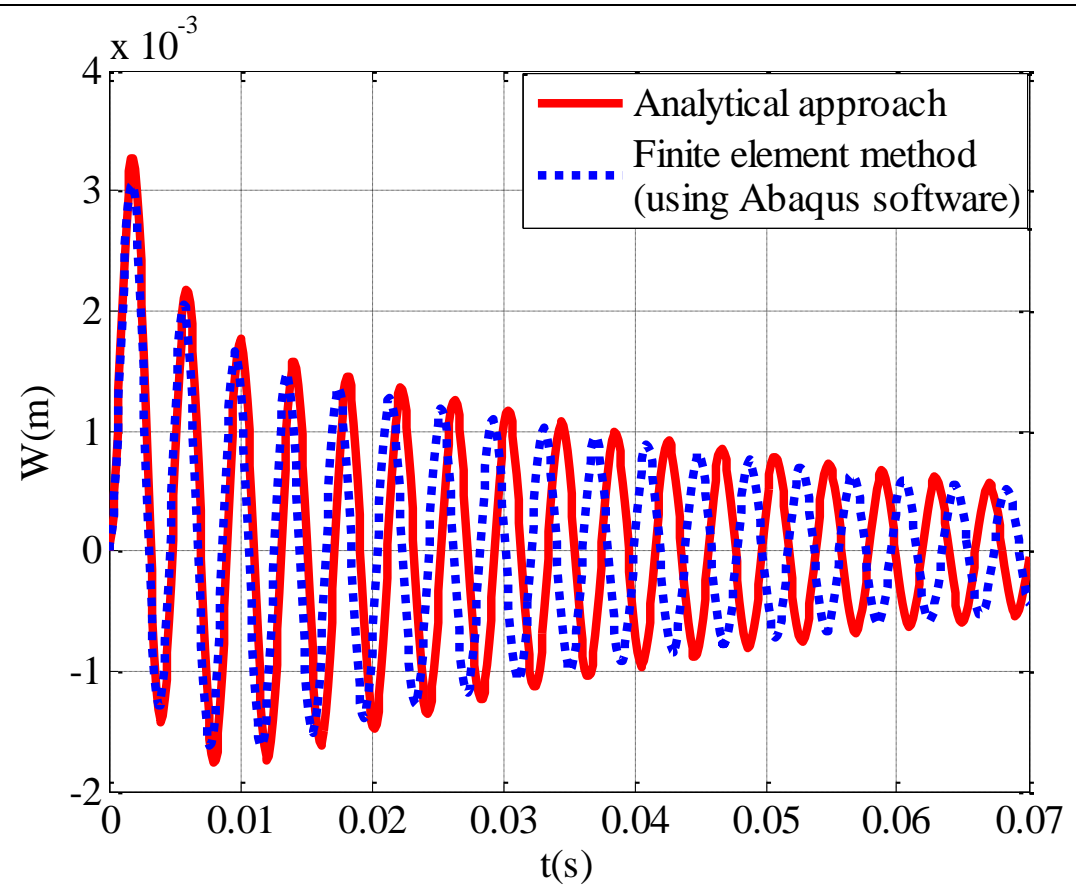

Fig. 2. Comparison of nonlinear dynamic response of perfect FGM plate without elastic foundations subjected to blast load. 


\section{ACCEPTED MANUSCRIPT}
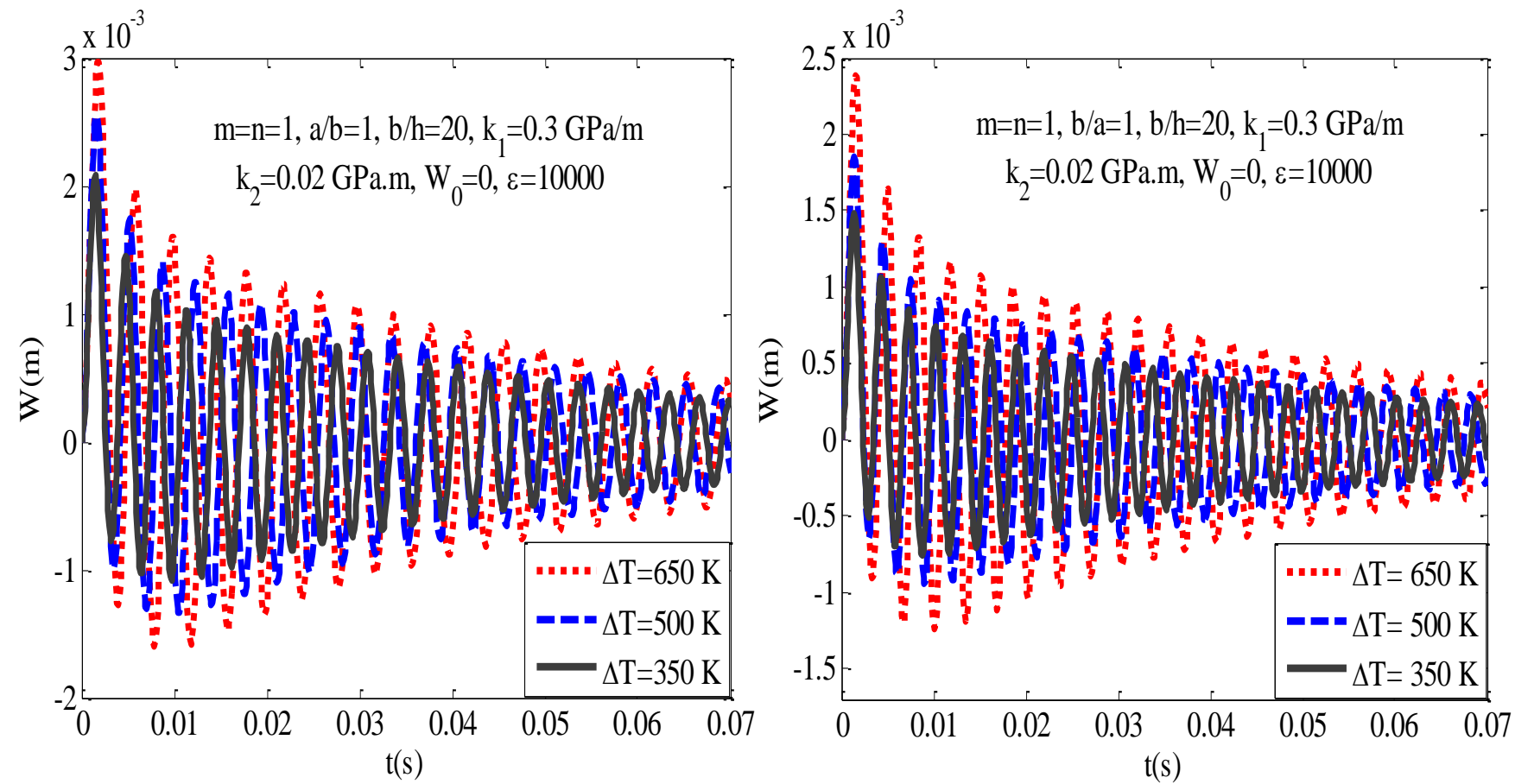

Fig. 3. Effects of temperature increment $\Delta T$ on Fig. 4. Effects of temperature increment $\Delta T$ on the nonlinear dynamic response of the simply the nonlinear dynamic response of the FGM plate supported FGM plate.

with two edges simply supported and two edges clamped. 


\section{ACCEPTED MANUSCRIPT}

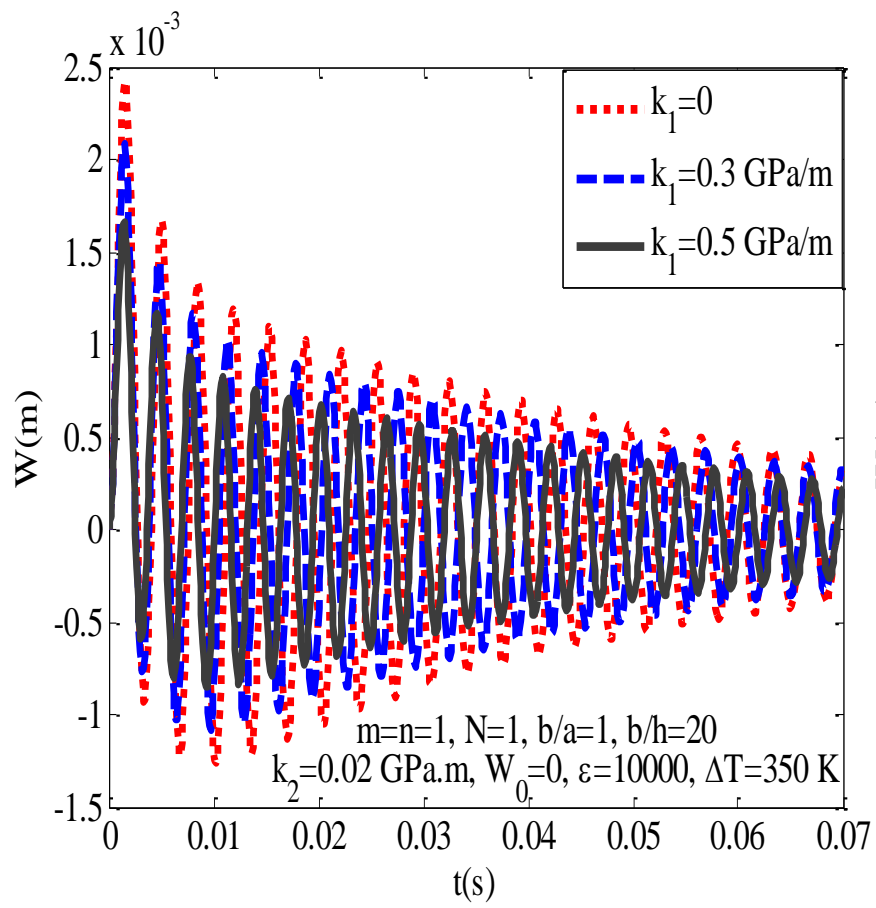

Fig. 5. Effect of the linear Winkler foundation on the nonlinear dynamic response of the simply supported FGM plate.

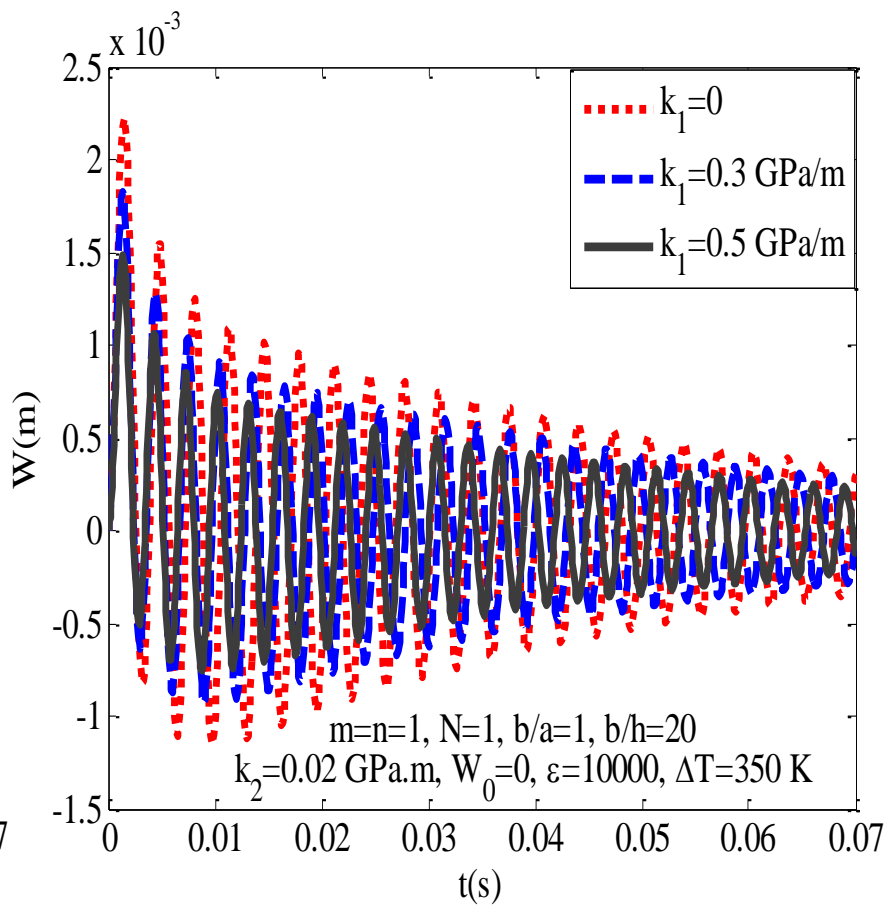

Fig. 6. Effect of the linear Winkler foundation on the nonlinear dynamic response of the FGM plate with two edges simply supported and two edges clamped. 


\section{ACCEPTED MANUSCRIPT}

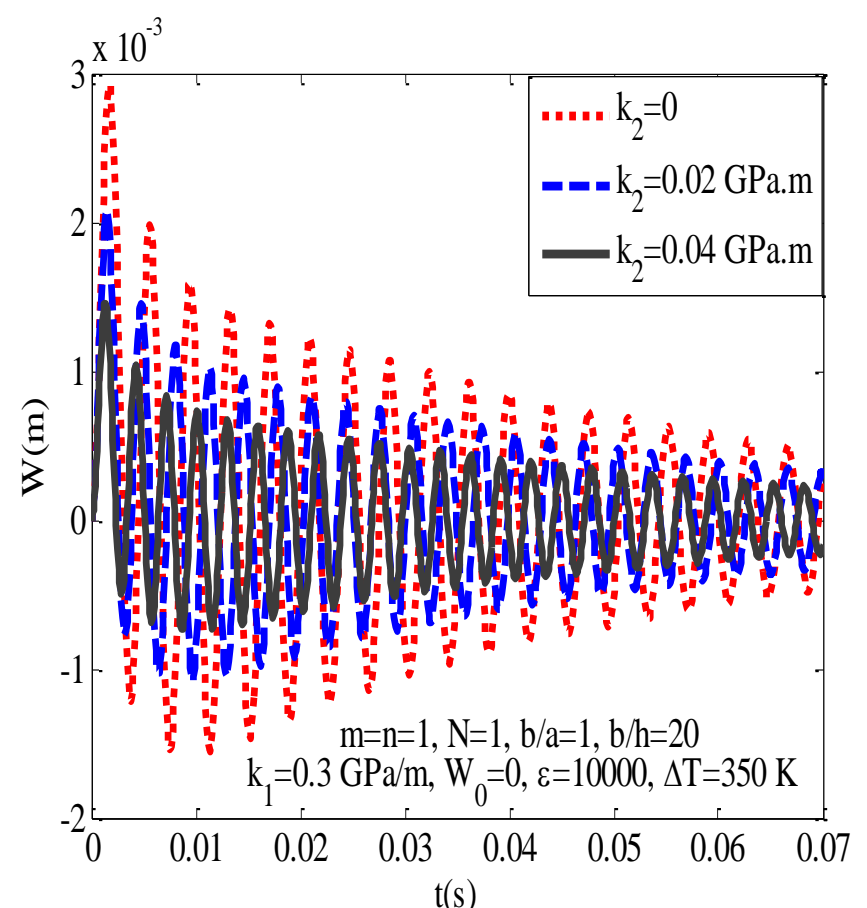

Fig. 7. Effect of the Pasternak foundation on the nonlinear dynamic response of the simply supported FGM plate.

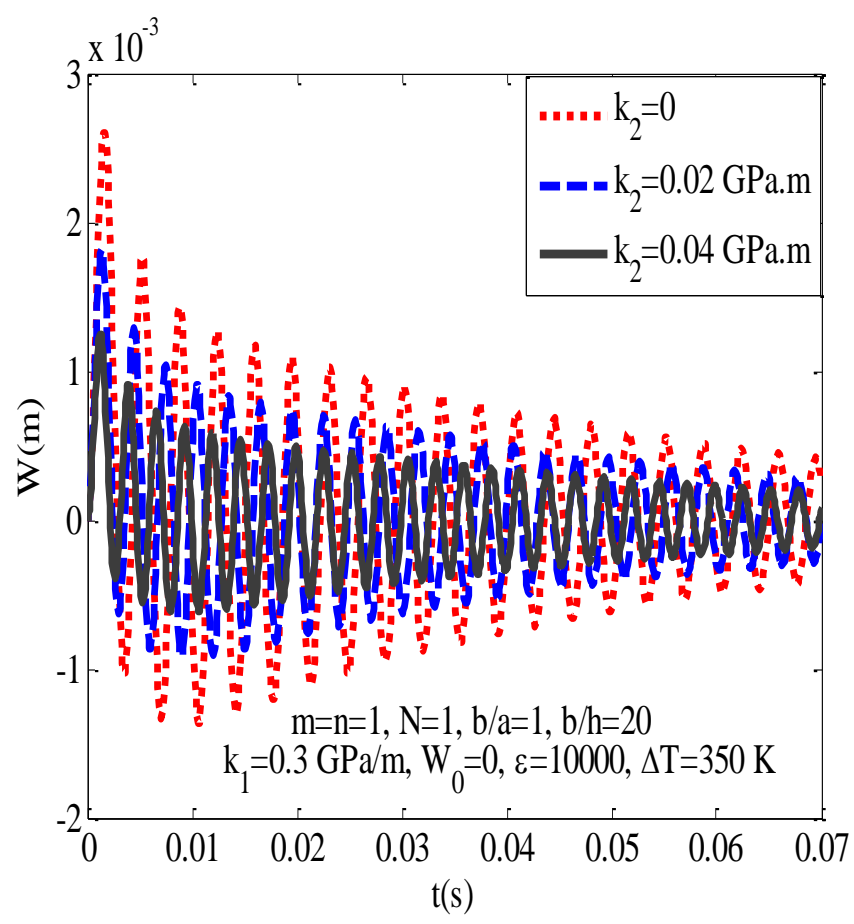

Fig. 8. Effect of the Pasternak foundation on the nonlinear dynamic response of the FGM plate with two edges simply supported and two edges clamped. 


\section{ACCEPTED MANUSCRIPT}

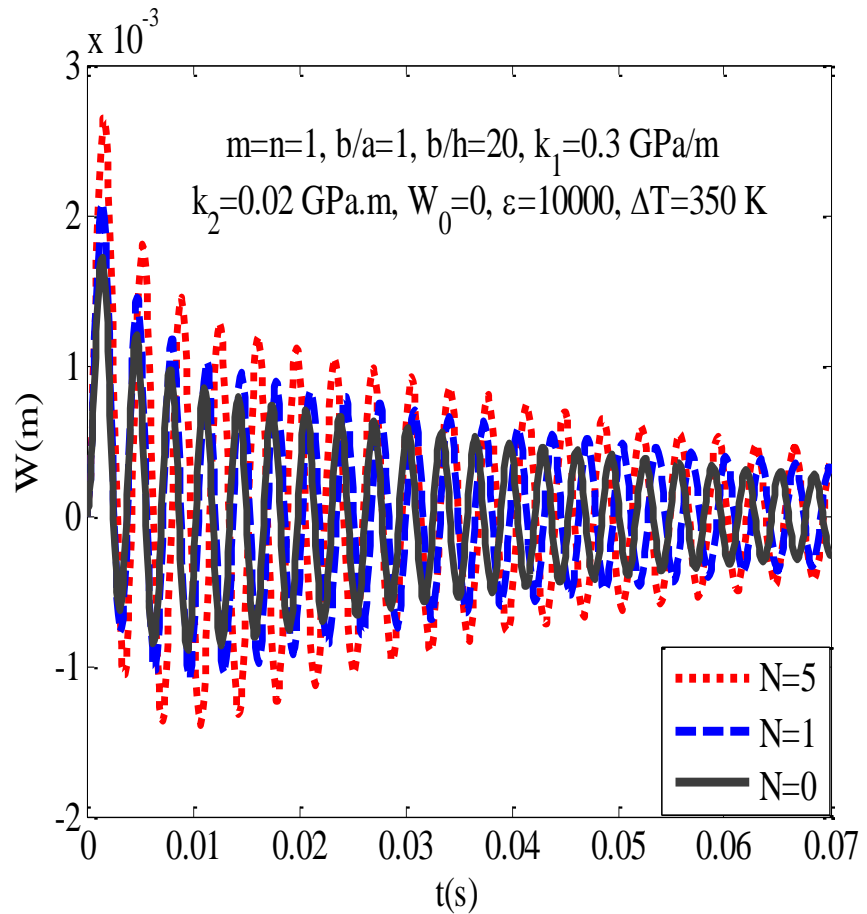

Fig. 9. Effect of volume fraction index $N$ on nonlinear dynamic response of the simply supported FGM plate.

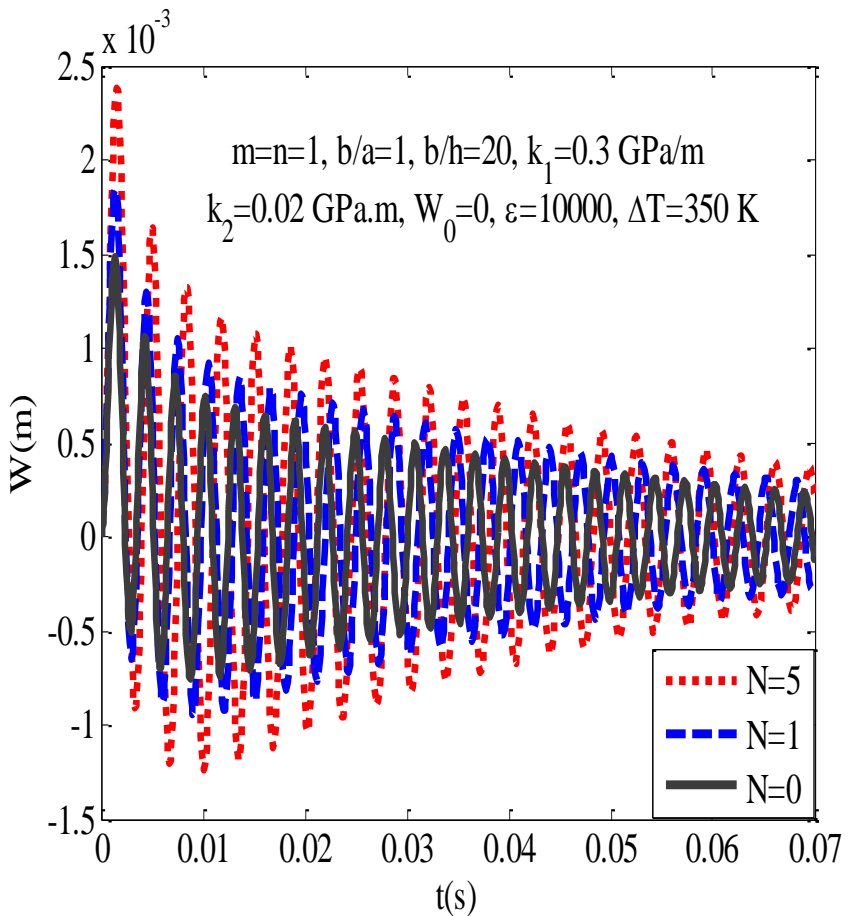

Fig. 10. Effect of volume fraction index $N$ on nonlinear dynamic response of the FGM plate with two edges simply supported and two edges clamped. 


\section{ACCEPTED MANUSCRIPT}

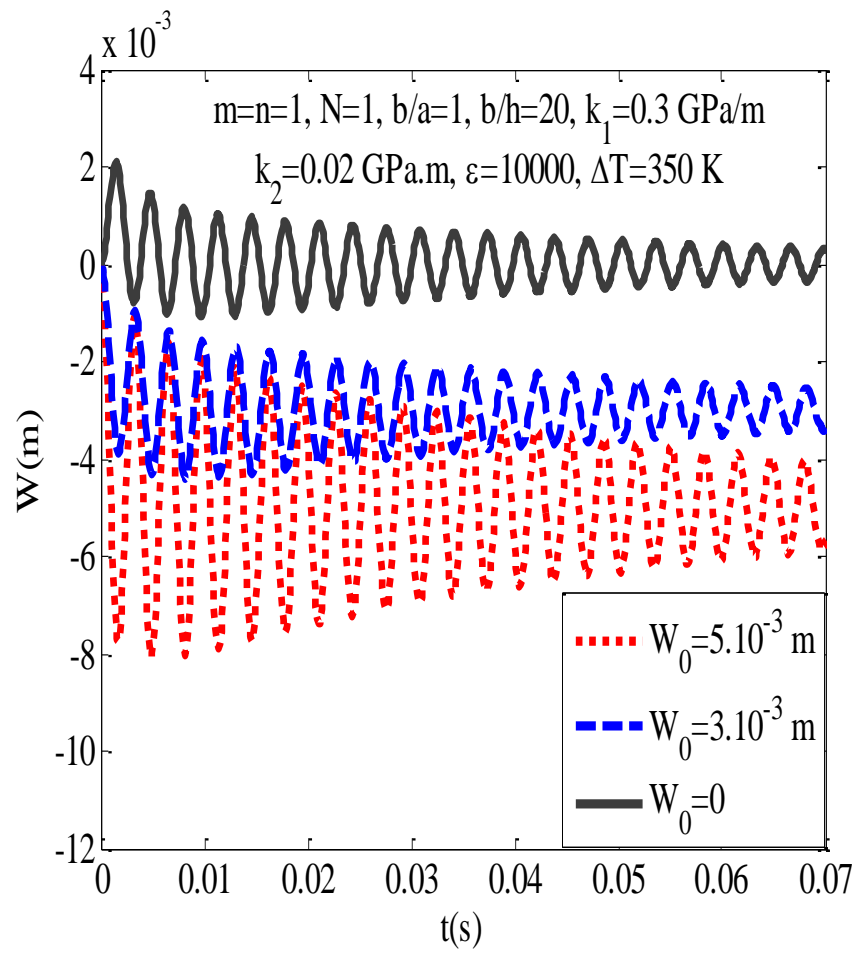

Fig. 11. Effects of initial imperfection on the nonlinear dynamic response of the simply supported FGM plate.

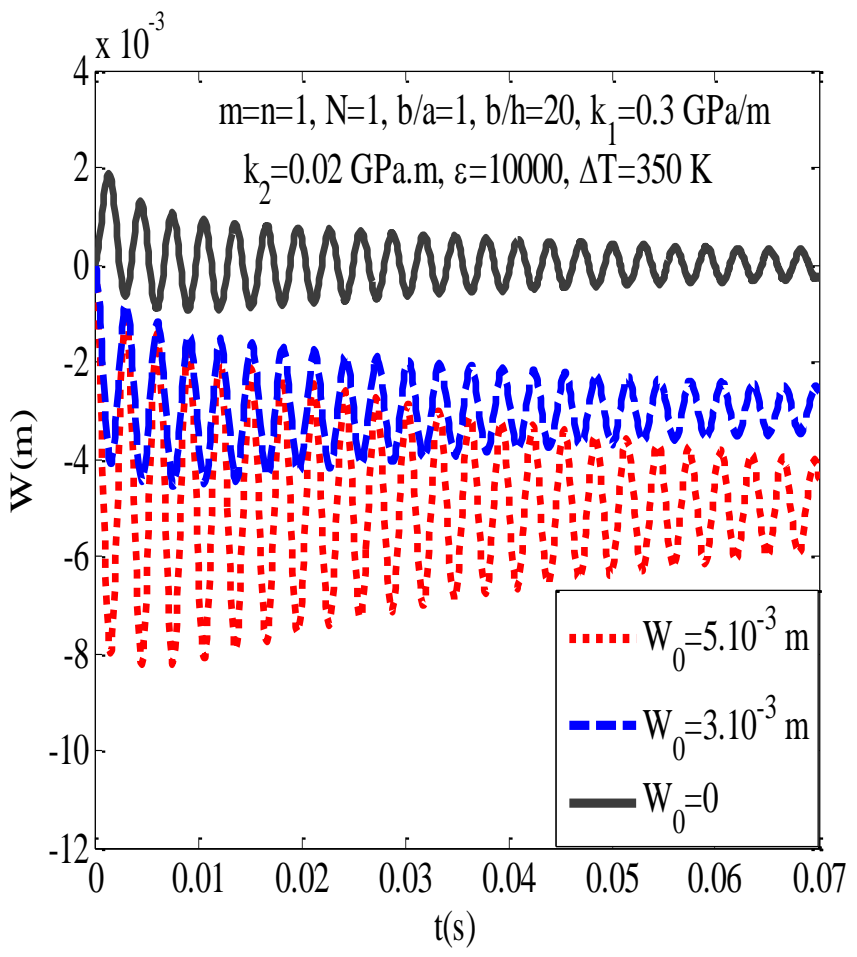

Fig. 12. Effects of initial imperfection on the nonlinear dynamic response of the FGM plate with two edges simply supported and two edges clamped. 


\section{ACCEPTED MANUSCRIPT}

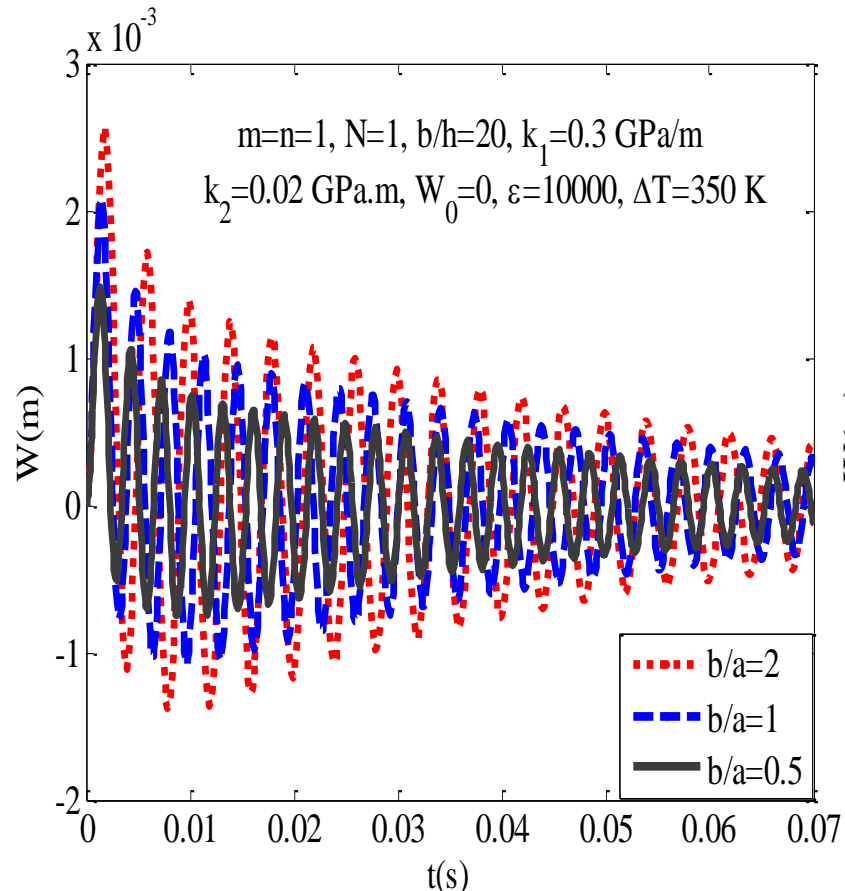

Fig. 13. Effects of ratio $b / a$ on the nonlinear dynamic response of the simply supported FGM plate.

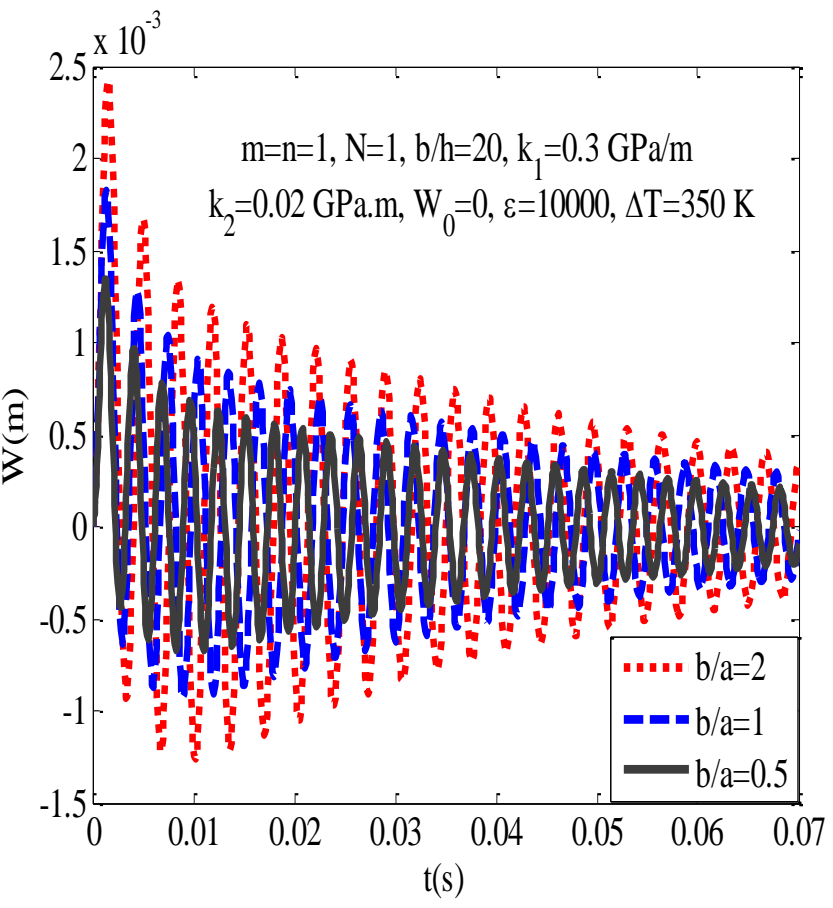

Fig. 14. Effects of ratio $b / a$ on the nonlinear dynamic response of the FGM plate with two edges simply supported and two edges clamped. 


\section{ACCEPTED MANUSCRIPT}

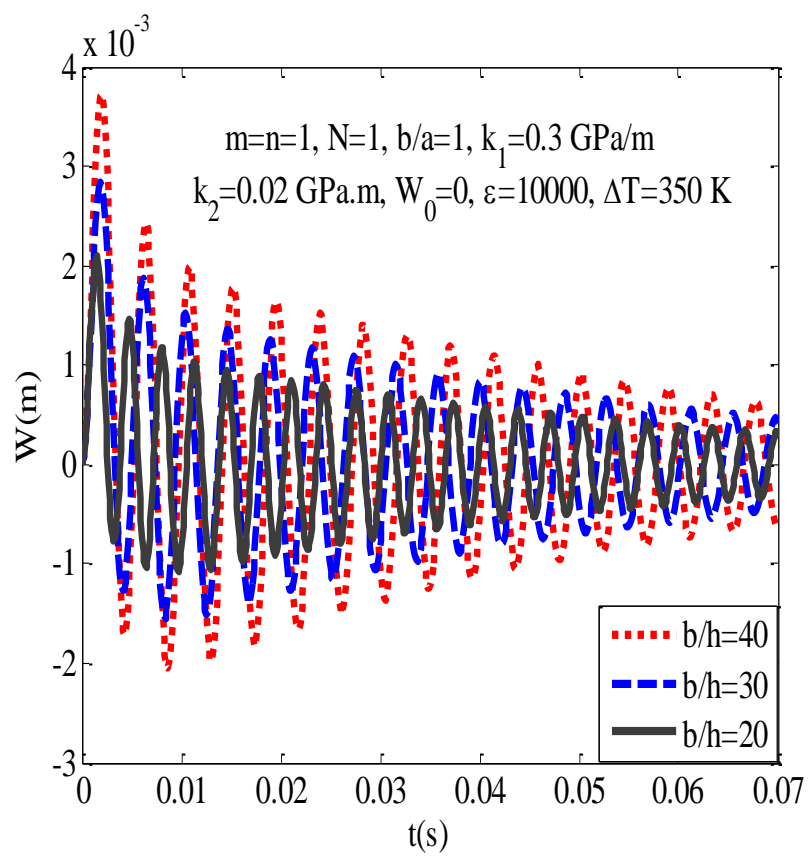

Fig. 15. Effects of ratio $b / h$ on the nonlinear dynamic response of the simply supported FGM plate.

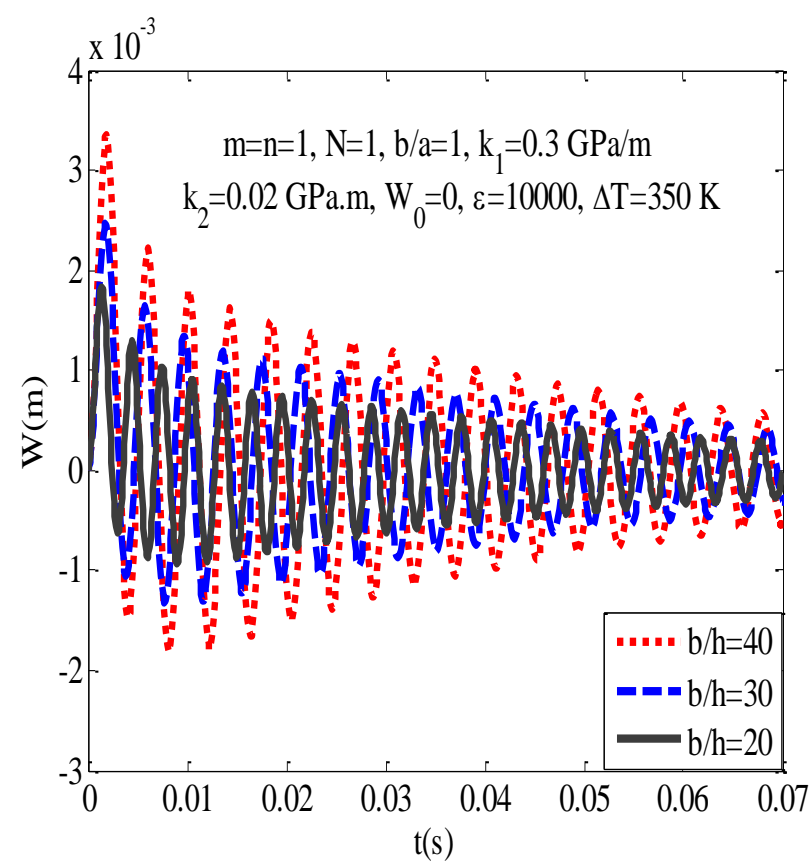

Fig. 16. Effects of ratio $b / h$ on the nonlinear dynamic response of the FGM plate with two edges simply supported and two edges clamped. 


\section{ACCEPTED MANUSCRIPT}

Table 1. Material properties of the constituent materials of the considered FGM plates.

\begin{tabular}{|c|c|c|c|c|c|c|}
\hline Material & Property & $P_{0}$ & $P_{-1}$ & $P_{1}$ & $P_{2}$ & $P_{3}$ \\
\hline \multirow{4}{*}{$\mathrm{Si}_{3} \mathrm{~N}_{4}$} & $\rho\left(\mathrm{kg} / \mathrm{m}^{3}\right)$ & 2370 & 0 & 0 & 0 & 0 \\
\cline { 2 - 7 }$($ Ceramic $)$ & $\alpha\left(K^{-1}\right)$ & $5.8723 \mathrm{e}-6$ & 0 & $9.095 \mathrm{e}-4$ & 0 & 0 \\
\cline { 2 - 7 } & $K(\mathrm{~W} / m K)$ & 13.723 & 0 & 0 & 0 & 0 \\
\cline { 2 - 7 } & $E(\mathrm{~Pa})$ & $201.04 \mathrm{e} 9$ & 0 & $3.079 \mathrm{e}-4$ & $-6.534 \mathrm{e}-7$ & 0 \\
\hline \multirow{3}{*}{ SUS304 } & $\rho\left(\mathrm{kg} / \mathrm{m}^{3}\right)$ & 8166 & 0 & 0 & 0 & 0 \\
\cline { 2 - 7 }$($ Metal $)$ & $\alpha\left(K^{-1}\right)$ & $12.330 \mathrm{e}-6$ & 0 & $8.086 \mathrm{e}-4$ & 0 & 0 \\
\cline { 2 - 7 } & & & & & & \\
\cline { 2 - 7 } & $K(\mathrm{~W} / m K)$ & 15.379 & 0 & 0 & 0 & 0 \\
\hline
\end{tabular}




\section{ACCEPTED MANUSCRIPT}

Table 2. Effects of temperature increment, $\Delta T$, and elastic foundations on the natural frequency $\left(s^{-1}\right)$ of the simply supported FGM plate with $m=n=1, b / a=1, b / h=20$.

\begin{tabular}{|c|c|c|c|c|}
\hline$\Delta T(K)$ & $N$ & $\left(k_{1}, k_{2}\right)=(0,0)$ & $\left(k_{1}, k_{2}\right)=(0.3,0.02)$ & $\left(k_{1}, k_{2}\right)=(0,0.04)$ \\
\hline \multirow{4}{*}{0} & 0 & 18423.2 & 28208.7 & 34629.3 \\
\hline & 1 & 10869.4 & 17732.5 & 22270.5 \\
\hline & 5 & 8790.7 & 14792.2 & 18752.6 \\
\hline & 10 & 8342.1 & 14227.9 & 18099.1 \\
\hline \multirow{4}{*}{500} & 0 & 17628.7 & 27194.3 & 33807.2 \\
\hline & 1 & 10095.9 & 17170.6 & 21825.8 \\
\hline & 5 & 8050.2 & 14351.8 & 18406.0 \\
\hline & 10 & 7602.5 & 13814.1 & 17777.9 \\
\hline \multirow{4}{*}{1000} & 0 & 16803.3 & 26666.8 & 33384.6 \\
\hline & 1 & 8053.6 & 16055.0 & 20959.5 \\
\hline & 5 & 5678.4 & 13168.4 & 17499.2 \\
\hline & 10 & 5266.9 & 12680.7 & 16910.4 \\
\hline
\end{tabular}




\section{University Library}

\section{- M M I N E R VA \\ A gateway to Melbourne's research publications}

Minerva Access is the Institutional Repository of The University of Melbourne

Author/s:

Nguyen, DD;Ngo, DT;Phuong, T;Tran, QQ

Title:

Nonlinear dynamic response and vibration of imperfect shear deformable functionally graded plates subjected to blast and thermal loads

Date:

2017-01-01

Citation:

Nguyen, D. D., Ngo, D. T., Phuong, T. \& Tran, Q. Q. (2017). Nonlinear dynamic response and vibration of imperfect shear deformable functionally graded plates subjected to blast and thermal loads. MECHANICS OF ADVANCED MATERIALS AND STRUCTURES, 24 (4), pp.318-329. https://doi.org/10.1080/15376494.2016.1142024.

Persistent Link:

http://hdl.handle.net/11343/59577 